\title{
Nuclear HMGB1 protects from non-alcoholic fatty liver diseases through negative regulation of liver $X$ receptor
}

Jean Personnaz ${ }^{1,2}$, Enzo Piccolo ${ }^{1,2}$, Alizée Dortignac ${ }^{1,2}$, Jason S. Iacovoni ${ }^{2}$, Jérôme Mariette ${ }^{3}$, Arnaud Polizzi ${ }^{4}$, Aurélie Batut ${ }^{2}$, Simon Deleruyelle ${ }^{2}$, Romain Paccoud ${ }^{2}$, Elsa Moreau ${ }^{2}$, Frédéric Martins $^{2,5}$, Thomas Clouaire ${ }^{6}$, Fadila Benhamed ${ }^{7}$, Alexandra Montagner ${ }^{2}$, Walter A. Wahli ${ }^{4,8,9}$, Robert F. Schwabe ${ }^{10}$, Armelle Yart ${ }^{1,2}$, Isabelle Castan-Laurell ${ }^{1,2}$, Catherine Postic ${ }^{7}$, Cédric Moro ${ }^{2}$, Gaelle Legube ${ }^{6}$, Chih-Hao Lee ${ }^{11}$, Hervé Guillou ${ }^{4}$, Philippe Valet ${ }^{1,2}$, Cédric Dray ${ }^{1,2} \&$ Jean10 Philippe Pradère ${ }^{1,2^{*}}$

${ }^{1}$ Institut RESTORE, UMR 1301, Institut National de la Santé et de la Recherche Médicale (INSERM), -CNRS-Université Paul Sabatier, Université de Toulouse, Toulouse, France.

${ }^{2}$ Institut des Maladies Métaboliques et Cardiovasculaires, UMR 1048/I2MC, Institut National de la Santé et de la Recherche Médicale (INSERM), Université de Toulouse, Toulouse, France.

${ }^{3}$ MIAT, Université de Toulouse, INRAE, 31326 Castanet-Tolosan, France.

${ }^{4}$ Toxalim, INRAE UMR 1331, ENVT, INP-Purpan, University of Toulouse, Paul Sabatier University, F-31027, Toulouse, France.

${ }^{5}$ Plateforme GeT, Genotoul, 31100 Toulouse, France.

${ }^{6}$ LBCMCP, Centre de Biologie Intégrative (CBI), CNRS, Université de Toulouse, France.

${ }^{7}$ Université de Paris, Institut Cochin, CNRS, INSERM, F- 75014 Paris, France.

${ }^{8}$ Center for Integrative Genomics, University of Lausanne, Le Génopode, CH-1015 Lausanne, Switzerland.

${ }^{9}$ Lee Kong Chian School of Medicine, Nanyang Technological University Singapore, Clinical Sciences Building, 11 Mandalay Road, Singapore 308232, Singapore.

${ }^{10}$ Department of Medicine, Columbia University, New York, New York, USA.

${ }^{11}$ Department of Molecular Metabolism, Harvard T.H. Chan School of Public Health, Boston, MA, USA

* Corresponding author: jean-philippe.pradere@inserm.fr

Running title: Nuclear HMGB1 as a new repressor of lipid synthesis in hepatocyte. 


\section{Abstract}

Dysregulations of lipid metabolism in the liver may trigger steatosis progression leading to potentially severe clinical consequences such as non-alcoholic fatty liver diseases (NAFLD). Molecular mechanisms underlying liver lipogenesis are very complex and fine-tuned by chromatin dynamics and the activity of multiple key transcription factors. Here, we demonstrate that the nuclear factor HMGB1 acts as a strong repressor of liver lipogenesis during metabolic stress in NAFLD. Mice with liver-specific Hmgbl-deficiency display exacerbated liver steatosis and hepatic insulin resistance when subjected to a high-fat diet or after fasting/refeeding. Global transcriptome and functional analysis revealed that the deletion of Hmgbl gene enhances LXR $\alpha$ activity resulting in increased lipogenesis. HMGB1 repression is not mediated through nucleosome landscape re-organization but rather via a preferential DNA occupation in region carrying genes regulated by LXR $\alpha$. Together these findings suggest that hepatocellular HMGB1 protects from liver steatosis development. HMGB1 may constitute a new attractive option to therapeutically target $\mathrm{LXR} \alpha$ axis during NAFLD. 


\section{Introduction}

Along the epidemic of obesity, non-alcoholic fatty liver disease (NAFLD) is progressing worldwide, affecting nearly $25 \%$ of the world-wide adult population (1) and generating numerous complications such as liver insulin resistance, non-alcoholic steatohepatitis and hepatocellular carcinoma (2). Liver steatosis consists in ectopic lipid storage within the hepatocytes, which aims at buffering circulating lipids and thus preventing lipotoxicity in different organs. Mechanisms underlying lipogenesis (from lipid uptake to lipid esterification and de novo lipogenesis) are extremely complex and consist in a subtle orchestration of the actions of different transcription factors (TFs) in close coordination with chromatin dynamics (3).

Among TFs involved in liver lipogenesis regulation, Liver X Receptors (LXRs) are members of the nuclear hormone receptor superfamily and are among the most central/dominant actors in this process. LXRs consist in two isotypes that share a very high homology but differ in their tissue expression profile. While LXR $\alpha(\mathrm{NR} 1 \mathrm{H} 3)$ is mainly expressed in metabolic tissues (liver, adipose tissues), LXR $\beta$ (NR1H2) is expressed ubiquitously (4). In the context of dyslipidemia or fasting/refeeding conditions and after activation by certain lipid species (5), LXRs directly coordinate, in a duo with its obligate partner, retinoic acid receptor (RXR), the expression of numerous key enzymes involved in cholesterol and lipid metabolism (Abcg5, Abcg8, Fasn, Scd1), but are also capable to modulate indirectly the lipogenesis through the regulation of other key TFs like SREBP1c, ChREBP or $\operatorname{PPAR} \gamma(4,6,7)$ that are also involved in the lipogenic transcription program. The current consensus on liver lipogenesis is that there is a hierarchical interplay between all TFs involved, where LXR is a very central piece; SREBP1 and ChREBP are crucial downstream key players while PPAR $\gamma$ 's role appears more supportive (8). LXRs activity is subtly regulated by the interaction with the nuclear receptor co-repressors $(\mathrm{NCoR})$ or the nuclear receptor coactivators protein complex (8) upon specific agonist activation. Recent evidences are now showing the emerging role of some methylase/demethylase enzymes in the modulation of LXR activity through the chromatin packaging and subsequent availability, adding one more complex layer of regulation $(9,10)$.

Global knockout of LXRs induces a severe reduction of liver lipid synthesis in wild type mice and could even prevent liver steatosis in ob/ob mice (11-13). LXR $\alpha$ deletion knockout leads to a drastic down-regulation of Srebfl expression associated with a reduced lipogenesis (6). Moreover LXRs agonist treatment increases plasma and hepatic TG in mice and humans $(14,15)$ supporting a key role of LXRs in fatty acid synthesis and liver steatosis progression. Therapeutic targeting of 
LXRs is still challenging as adverse effects have been described (15) and more insights regarding LXRs upstream regulators may be helpful to design novel therapeutic avenues.

HMGB1 belongs to the family of high mobility group proteins, which after the histones represents the most abundant proteins in the nucleus. In recent years, HMGB1 has also been scrutinized for its role in the extracellular cellular compartment as a potent inflammatory factor, notably during sterile inflammation (9). Originally however, HMGB1 has been known for its role in the nucleus (17) as a protein capable of binding chromatin on unspecific domains (18) in a very dynamic manner (19). HMGB1 may affect several biological functions such as VDJ recombination, DNA repair (20), chromatin assembly and gene transcription through different mechanisms, such as DNA bending/looping, nucleosome formation $(21,22)$, interaction with the transcription machinery including TFs themselves $(19,23-25)$. A very recent report depicts nuclear HMGB1 as an even more versatile factor able to bind to topologically-associated domains or RNA directly to regulate proliferation or senescence programs (26). In cultured cells, while HMGB1 deletion leads to minor changes in histone numbers, it results in notable changes of the RNA pool (22), in local chromatin remodeling (27) or the global transcriptome (26). However only a sparse number of studies have been carried out in vivo (27). The global ablation of Hmgbl generates a severe phenotype with perinatal mortality (28), likely due to a defective glucocorticoid signaling leading to a poor utilization of hepatic glycogen and resulting in a lethal hypoglycemia, whereas hepatocyte-specific HMGB1 ablation did not have a major impact under homeostatic conditions (29). Thus, in this context it seems particularly relevant to explore the role of nuclear HMGB1 in vivo especially during metabolic stress, where the dynamics of the chromatin are critical to orchestrate the activity of key TFs and gene transcription programs in order to buffer stress mediators and maintain whole-body homeostasis.

Here, we unveiled the important role of HMGB1 in the repressive effect of LXRs, in particular LXR $\alpha$, during metabolic stress, as demonstrated by increased liver steatosis and an alteration of the hepatic insulin in hepatocyte-specific Hmgbl knockout $\left(\mathrm{HMGB}^{\Delta \mathrm{Hep}}\right)$ mice subjected to either a high-fat diet (HFD) or a fasting-refeeding ( F/R) challenge. In vitro assays further confirmed the repressive action that HMGB1 exerts on $\mathrm{LXR} \alpha$ activity. Taken together, our data reveal a novel role of HMGB1 in alleviating liver steatosis through the repression of LXR $\alpha$ during metabolic stress. 


\section{Results}

\section{Hepatic deletion of Hmgb1 increases liver steatosis during metabolic stress.}

$H m g b 1$ hepatocyte-specific knockout mice (HMGB1 ${ }^{\Delta \mathrm{Hep}}$ ) under chow diet (CD) feeding display no major changes in liver transcriptome and no drastic phenotype of glycogen utilization compared to control mice $\left(\mathrm{HMGB}^{\mathrm{fl} / \mathrm{fl}}\right)$ (29), contrasting findings from the global Hmgbl knockout on metabolism, possibly due to particular functions during development (28). This prompted us to clarify the precise function of HMGB1 in liver metabolism by studying the role of HMGB1 as a potential regulator of global and/or hepatic energy metabolism in adult mice using a careful characterization of $H M G B 1^{\mathrm{fl} / \mathrm{fl}}$ and $\mathrm{HMGB} 1^{\Delta \mathrm{Hep}}$ mice subjected to metabolic stress. A complete metabolic checkup in adult mice upon CD, showed that deletion of Hmgbl in hepatocytes (fig. S1A) did not affect circulating levels of HMGB1 (fig. S1B), serum liver enzyme levels (fig. S1C), body weight (fig. S1D), lean/fat mass ratio (fig. S1E) fasting blood glucose levels and glucose homeostasis (fig. S1F) nor generated any changes in hepatic lipid contents (fig. S1G) or in food consumption and other parameters assessed by indirect calorimetry (not shown). However, a high-throughput real-time qPCR gene expression profiling targeting metabolic pathways revealed that many key genes involved in lipid metabolism and lipogenesis, such as Cd36, Fasn or Acly, were upregulated in the liver of HMGB1 ${ }^{\Delta \mathrm{Hep}}$ mice compared to $\mathrm{HMGB}^{\mathrm{fl} / \mathrm{fl}}$ mice (fig. S1H). Collectively, these data suggest, while supporting conclusions from a previous report (29) on the minor role of HMGB1 in systemic and liver metabolic homeostasis, that its function might become relevant in the setting of metabolic stress. To test this hypothesis, HMGB1 ${ }^{\mathrm{fl} / \mathrm{fl}}$ and $\mathrm{HMGB} 1^{\Delta \mathrm{Hep}}$ mice were subjected to a high-fat diet feeding (HFD60\%). After 12 weeks of this regimen, HMGB $1^{\mathrm{fl} / \mathrm{fl}}$ control mice showed the expected weight gain and glucose metabolism deterioration compared to mice fed CD (not shown). In this context, after HFD60\%, both genotypes displayed similar weight gain (fig. S2A) and similar fat mass (fig. S2B) and shared identical physiological parameters (food intake, respiratory quotient, physical activity) (fig. S2C-E). However, HMGB1 ${ }^{\Delta \mathrm{Hep}}$ mice exhibited a significant increase in Oil Red-O staining (Fig. 1A) and in liver lipid content, especially cholesterol ester, compared to control mice (Fig. 1B). In addition, mRNA expression analysis revealed a drastic upregulation of key genes involved in liver lipid metabolism and lipogenesis such as Cd36, Fasn, Scd-1, Pnpla3, Adrp47 or Lxra (Fig. 1C) in livers from HMGB1 ${ }^{\Delta \mathrm{Hep}}$ mice compared to control littermates. To further challenge the lipogenic pathway using a more acute nutritional setting without confounding effects related to a 12 week-HFD, HMGB1 $1^{\mathrm{fl} / \mathrm{fl}}$ and $\mathrm{HMGB}^{\Delta \mathrm{Hep}}$ mice were subjected to a 6 hour-fast and an 8 hour-chow diet refeeding (F/R) experiment. Similar to HFD, hepatic lipid accumulation in $\mathrm{HMGB}^{\triangle \mathrm{Hep}}$ mice was notably more 
pronounced compared to control mice, as supported by a drastic increase of Oil Red-O staining on liver sections (Fig. 1D), of hepatic lipid levels (Fig. 1E) and lipogenic gene expression (Fig. 1F) in liver biopsies from $H M G B 1^{\Delta \mathrm{Hep}}$ mice compared to $H M G B 1^{\mathrm{fl} / \mathrm{fl}}$ mice. To confirm the $H M G B 1^{\Delta \mathrm{Hep}}$ mice phenotype, several other diets designed to challenge the hepatic lipogenesis were implemented, such as 24 week HFD, 8 week-choline deficient-HFD and a 12 week-high fat-high fructose diet, all showing a consistent and more severe liver steatosis in $\mathrm{HMGB}^{\Delta \mathrm{Hep}}$ mice compared to $\mathrm{HMGB} 1^{\mathrm{fl} / \mathrm{fl}}$ mice (fig. S3A-C). These results indicate that under several steatosis-promoting regimens, $\mathrm{Hmgbl}$ deletion in hepatocytes is associated with a more active liver lipogenesis, suggesting that HMGB1 might play a repressive role on liver lipid synthesis, thereby preventing steatosis.

\section{Nuclear HMGB1 represses hepatocyte lipogenesis in vivo and in vitro in a cell-autonomous} manner.

The enhanced hepatosteatosis in $\mathrm{HMGB}^{\Delta \mathrm{Hep}}$ mice may result from an increased activity of lipogenesis in the hepatocytes. To address this question, hepatic lipid synthesis was monitored in vivo using radiolabeled substrates upon a fasting-refeeding challenge (Fig. 2A). After 6 hours of fasting, $\mathrm{HMGB} 1^{\mathrm{fl} / \mathrm{fl}}$ and $\mathrm{HMGB} 1^{\Delta \mathrm{Hep}}$ mice received a bolus of ${ }^{3} \mathrm{H}$ glucose, and the ${ }^{3} \mathrm{H}$ radioisotope incorporation was quantified in the lipid fractions of several tissues after 8 hours of refeeding. Upon $\mathrm{CD}$, while $\mathrm{F} / \mathrm{R}$ induced a strong ${ }^{3} \mathrm{H}$ incorporation mainly in brown adipose tissue (BAT) and liver of HMGB $1^{\mathrm{fl} / \mathrm{fl}}$ mice (Fig. 2A), this effect was even more pronounced in HMGB1 ${ }^{\Delta \mathrm{Hep}}$ mice, suggesting a higher capacity of Hmgbl-null hepatocytes to synthesize lipids after refeeding (Fig. 2A). In parallel, we evaluated in vivo, a potential disturbance of lipoprotein metabolism in HMGB1 ${ }^{\Delta \mathrm{Hep}}$ mice upon CD and HFD. The VLDL secretion after treatment with the lipoprotein lipase inhibitor tyloxapol (Fig. 2B) and the activity of the microsomal triglyceride transfer protein (MTP), a key enzyme involved in lipid export (Fig. 2C), were both identical in $\mathrm{HMGB}^{\mathrm{fl} / \mathrm{fl}}$ and $\mathrm{HMGB} 1^{\Delta \mathrm{Hep}}$ mice subjected to CD and HFD.

Present knowledge indicates that the regulation of hepatic lipogenesis depends on the interplay, within the liver, between hepatocytes and non-parenchymal cells and is also influenced by other tissues, mainly the adipose tissue. Therefore, we interrogated whether the increase of liver lipogenesis in $\mathrm{HMGB}^{\Delta \mathrm{Hep}}$ mice could be cell-autonomous. To address this point, primary hepatocytes were isolated from $\mathrm{HMGB} 1^{\mathrm{fl} / \mathrm{fl}}$ and $\mathrm{HMGB} 1^{\Delta \mathrm{Hep}}$ mice and lipogenic activity was assessed in vitro using the same strategy as described above for the in vivo study. Consistent with the in vivo data, after isolation from mice under CD, cultured HMGB ${ }^{\Delta \mathrm{Hep}}$ hepatocytes displayed an increased lipogenic activity compared to HMGB1 $1^{\mathrm{fl} / \mathrm{fl}}$ hepatocytes (Fig. 2D). 
However, lipogenesis was stimulated to the same extent by insulin (Fig. 2D) in hepatocytes from both genotypes. Interestingly, when isolated from HFD-fed mice, HMGB1 ${ }^{\triangle \mathrm{Hep}}$ hepatocytes still exhibited a higher lipogenic activity compared to HMGB1 $1^{\mathrm{fl} / \mathrm{fl}}$ hepatocytes (Fig. 2E) and insulin slightly increased the lipogenesis independently of the genotypes. Importantly, palmitate oxidation was also measured in primary hepatocytes from both genotypes, and no difference in lipid utilization was observed neither upon CD (Fig. 2F) nor HFD (Fig. 2G). Collectively, these results suggest that HMGB1 represses lipogenesis in hepatocytes in a cell autonomous-manner, without affecting FA oxidation.

\section{Hepatic deletion of Hmgb1 affects specifically liver insulin sensitivity.}

Studies have reported a strong correlation between hepatic lipid accumulation and a decreased insulin sensitivity in the liver (30). Therefore, we next monitored whether the liver steatosis induced by hepatocyte Hmgbl deletion has any effect on glucose homeostasis and/or insulin signaling in mice subjected to a HFD60\%. Upon HFD both $\mathrm{HMGB}^{\mathrm{fl} / \mathrm{fl}}$ and $\mathrm{HMGB} 1^{\Delta \mathrm{Hep}}$, displayed a similar glucose homeostasis and global insulin sensitivity (Fig. 3A-C), albeit a slight trend toward a higher AUC after oral glucose test tolerance was observed in HMGB1 ${ }^{\Delta \mathrm{Hep}}$ mice (Fig. 3A). Of note, insulin levels either after starvation or after a bolus of glucose were similar between both groups (Fig. 3B), ruling out that hepatic Hmgbl deletion may interfere with insulin secretion. Interestingly $\mathrm{HMGB}^{\Delta \mathrm{Hep}}$ mice displayed a higher glycaemia after 14 hours starvation (Fig. 3D), corroborated by a higher AUC during a pyruvate tolerance test compared to $\mathrm{HMGB}^{\mathrm{fl} / \mathrm{fl}}$ mice (Fig. 3E), suggesting an increased hepatic glucose production consistent with a potential hepatic insulin resistance. In addition, liver glycogen content was lower in $\mathrm{HMGB}^{\triangle \mathrm{Hep}}$ mice as shown by the PAS coloration (Fig. 3F-G) supporting a compromised glycogen synthesis. All together these data show that the increased hepatosteatosis in $\mathrm{HMGB} 1^{\Delta \mathrm{Hep}}$ mice is associated with a noticeable perturbation of insulin signaling. This was confirmed by the lower level of AKT phosphorylation, recognized as a classic downstream effector of the insulin receptor, in the liver of $\mathrm{HMGB}{ }^{\Delta \mathrm{Hep}}$ mice subjected to a 12 week-HFD, compared to $\mathrm{HMGB} 1^{\mathrm{fl} / \mathrm{fl}}$ mice (Fig. 3H). To functionally test a possible alteration of insulin sensitivity in absence of hepatocyte $H m g b 1, \mathrm{HMGB}^{\mathrm{fl} / \mathrm{fl}}$ and $\mathrm{HMGB} 1^{\Delta \mathrm{Hep}}$ mice subjected to CD or a long term HFD (24 weeks- as the global insulin signaling is more perturbed compared to a 12 week-HFD) were challenged with an acute injection of insulin $(0.75 \mathrm{U} / \mathrm{kg}$ ) or saline (Fig. 3I). In CD-fed mice of both genotypes, we observed no differences in the insulin-induced phosphorylation of AKT, compared to saline conditions (fig. S2F). In HFDfed mice, insulin injection induced the expected phosphorylation of AKT in the liver, adipose 
tissue and skeletal muscle (Fig. 3I) in control mice, but remarkably the amount of p-AKT was much lower selectively in liver samples harvested from HMGB1 ${ }^{\Delta \mathrm{Hep}}$ mice, compared to skeletal muscle and adipose tissue (Fig. 3I). Collectively these data show a selective impact of hepatocellular HMGB1 deficiency on liver insulin signaling upon long term-HFD feeding.

\section{The signaling of LXR is enhanced in the absence of Hmgb1.}

To unveil the signaling pathways regulated by HMGB1, we performed gene expression profiling using cDNA microarray of $\mathrm{HMGB}^{\mathrm{fl} / \mathrm{fl}}$ and $\mathrm{HMGB} 1^{\Delta \mathrm{Hep}}$ liver samples from mice subjected to a 12 week-HFD regimen or a F/R challenge (Fig. 4). Microarray analysis and unsupervised clustering displayed on the heatmaps showed that deletion of Hmgbl caused significant changes in the liver transcriptome (Fig. 4A, fig. S4A-B). Venn diagrams revealed that in liver samples from HMGB $1^{\Delta \mathrm{Hep}}$ mice, there were 295 up- and 471 down-regulated genes upon HFD and 125 up- and 380 down-regulated genes after F/R (Fig. 4B). Of note, as displayed in the Venn diagram (Fig. 4B), 253 genes (roughly 25\%) of the identified genes are similarly regulated in both challenges (HFD and F/R). Hierarchical clustering method showed that the vast majority of these genes are subjected to the same type of variations in both conditions (Fig. 4C) suggesting that these groups of genes belong to pathways under robust regulation by Hmgb1. The enrichment analysis of these 253 common genes, using the EnrichR database, indicated that among all gene ontology $(\mathrm{GO})$ terms represented in HMGB1 ${ }^{\triangle \mathrm{Hep}}$ livers, the most enriched GO terms were "metabolism of lipids" and "metabolism" (Fig. 4D-E), confirming our histological findings. Based on the analysis of the gene network using the Reactome database, numerous genes regulated by HMGB1 in both nutritional conditions, are connected to metabolism functions, and more specifically, to lipid metabolism (Fig. 4F). We then narrowed our focus on gene clusters involved in these identified GO terms, and further performed analysis on potential upstream regulators involved, by using EnrichR database (Fig. 4G). Interestingly, among the identified transcription factors, LXR and PPAR $\gamma$ came up with the highest score. LXR $\alpha$ and PPAR $\gamma$ are well known for their critical pro-lipogenic activity in the liver, which is in line with the phenotype displayed by the HMGB1 $1^{\Delta \mathrm{Hep}}$ mice (Fig. 1).

Collectively our unbiased transcriptomic study indicated that in the liver upon metabolic stress, HMGB1 might repress the expression of gene clusters partly controlled by LXR $\alpha$ and PPAR $\gamma$ and involved in hepatic lipid synthesis. 


\section{Exaggerated hepatic steatosis in the Hmgb1-null liver is dependent on LXRa activity.}

As LXR $\alpha$ is a key lipogenic transcription factor involved in cholesterol metabolism and liver lipogenesis, the potential de-repression of its activity induced by HMGB1 deletion could translates into liver steatosis $(31,32)$. However, it is less clear whether PPAR $\gamma$ is a significant trigger of liver steatosis. The role of PPAR $\gamma$ in HFD-induced hepatosteatosis is supported by several reports $(33,34)$, but no studies have investigated its potential role during F/R-induced liver steatosis. To clarify this, we subjected mice carrying hepatocyte specific-Ppary deletion to F/R challenge, and the results show no major contribution of hepatocyte PPAR $\gamma$ to the progression of F/R-induced liver steatosis (fig. S5) based on liver body weight ratio, Oil Red-O staining, neutral lipid profile or mRNA expression of hepatic steatosis markers (fig. S5A-D). This suggests that PPAR $\gamma$, per se, is not a determinant trigger of hepatic lipogenesis, and therefore its potential contribution in the severe steatosis displayed in HMGB1 ${ }^{\Delta \mathrm{Hep}}$ mice is likely minor.

Subsequently, we focused on the functional interdependence between HMGB1 and LXR $\alpha$, examining the effect of pharmacological activation and adenovirally-mediated inhibition of LXR $\alpha$ in HMGB $1^{\mathrm{fl} / \mathrm{fl}}$ and HMGB $1^{\Delta \mathrm{Hep}}$ mice (Fig. 5). To establish a possible causal link between the absence of HMGB1 and LXR $\alpha$ activity, the HMGB1 $1^{\mathrm{fl} / \mathrm{fl}}$ and $\mathrm{HMGB} 1^{\Delta \mathrm{Hep}}$ mice were treated with a synthetic LXR agonist (T0901317) for four consecutive days (30mg/kg-per os) (Fig. 5AB, fig. S6A). Remarkably, already before treatment, several LXR $\alpha$ dependent genes (Srebf1, Fasn, Elovl-6, Abcg5, and Abcg-8) were up-regulated in the HMGB1 ${ }^{\Delta H e p}$ livers (Fig. 5A). As expected, T0901317 treatment of $\mathrm{HMGB}^{\mathrm{fl} / \mathrm{fl}}$ mice potently induced expression of LXR dependent genes (Srebf1, Fasn, Elovl-6, Scd-1, Abcg5, Abcg-8) in the liver compared to vehicle treated $\mathrm{HMGB} 1^{\mathrm{fl} / \mathrm{fl}}$ mice. Importantly, $\mathrm{HMGB} 1^{\Delta \mathrm{Hep}}$ livers displayed a significantly higher response to T0901317 than HMGB1 ${ }^{\mathrm{fl} / \mathrm{fl}}$ mice, with an enhanced expression of Fasn, Elovl-6, Abcg-5 and Abcg-8 (Fig. 5A and fig. S6A). This higher response was corroborated by histological examination showing an increased Oil Red-O staining in Hmgbl deleted livers in mice subjected to the T0901317 treatment (Fig. 5B). Taken together these results indicate that the higher lipogenesis in HMGB1 ${ }^{\Delta \mathrm{Hep}}$ livers is likely due to an enhanced LXR $\alpha$ activity. To complement this study, and firmly establish the role of LXR $\alpha$ in the enhanced hepatic steatosis seen in $\mathrm{HMGB}^{\Delta \mathrm{Hep}}$ mice, we silenced LXR $\alpha$ expression in vivo, using an adenovirus expressing shRNA targeting the receptor (Ad-ShLxro) (Fig. 5C-D and fig. S6B-C). Seven days after viral infection, the hepatic $\operatorname{LXR} \alpha$, but not $\beta$, mRNA levels were reduced showing that expression of $\operatorname{LXR} \alpha$, as long as $\operatorname{LXR} \alpha$-dependent genes, were successfully blunted in AdShLxr $\alpha$ injected animals compared to control animals injected with an adenovirus expressing a 
scrambled shRNA (Ad-ShSCR), highlighting the potency and specificity of LXR $\alpha$ targeting (fig. S6B-C). Consistent with the results presented above, Ad-shSCR treated HMGB1 ${ }^{\Delta \mathrm{Hep}}$ mice displayed increased hepatic steatosis compared to Ad-shSCR injected HMGB1 ${ }^{\mathrm{fl} / \mathrm{fl}}$ mice either upon F/R (Fig. 5C) or HFD feeding (Fig. 5D) as shown by Oil Red-O staining. And remarkably, Ad-shLxr $\alpha$ treatment lowered drastically hepatic steatosis in both groups of animals (Fig. 5C-D), suggesting that LXR $\alpha$ plays a major role in the enhanced hepatic lipid synthesis of $H M G B 1^{\Delta H e p}$ mice. In summary, these results suggest that the LXR $\alpha$ activity is responsible for the enhanced hepatic lipid synthesis in Hmgbl-null livers and support a repressive role of HMGB1 on hepatic lipogenesis through repression of LXR $\alpha$ activity.

\section{HMGB1 binds to LXRa target genes involved in lipogenesis.}

Having identified LXR $\alpha$ as potential targets for repression by HMGB1, we determined the molecular mechanisms by which HMGB1 is exerting this action. Considering the impact HMGB1 may have on chromatin compaction (22), we first performed an assay for transposaseaccessible chromatin using high throughput sequencing (ATAC-seq) to evaluate the global chromatin dynamics in the absence of hepatic-HMGB1. Hepatocyte nuclei were purified from liver samples harvested from $\mathrm{HMGB} 1^{\mathrm{fl} / \mathrm{fl}}$ and $\mathrm{HMGB} 1^{\Delta \mathrm{Hep}}$ mice upon $\mathrm{CD}$ feeding or after FR (fig. S7). Remarkably, at basal state the principal component analysis (PCA) analysis of the ATAC-seq peaks revealed no distinct pattern in chromatin states between both genotypes (fig. $\mathrm{S} 7 \mathrm{~A}$ ), in reads alignment in a genome browser (fig. S7B) or in the open chromatin regions (OCR) locations around transcription start sites (TSS) (fig. S7C). In sharp contrast, F/R in $\mathrm{HMGB} 1^{\mathrm{fl} / f \mathrm{l}}$ mice triggered significant changes in chromatin state compared to the $\mathrm{CD}$ condition (respectively 68776 vs. 47725 OCRs), but similar modifications were detected in the liver chromatin from F/R HMGB1 ${ }^{\Delta \mathrm{Hep}}$ mice. Strikingly, only 4 OCRs were differentially nucleosome-depleted between both genotypes supported by the very high number of common aligned peaks (fig. S7D). PCA analysis, examination of TSS charts and annotation chart-pie confirmed the high similarity in the chromatin state of both librairies (fig. S7E-G). A close visualization of aligned peaks in loci of lipogenic genes regulated by LXR $\alpha$ (Srebfl, Scd-1, Cidec or Fasn) (fig. S7H) showed as expected the same chromatin state pattern between both genotypes. As presumed from this very low number of sites differentially opened in the chromatin between control and Hmgbl null-livers, enrichment analysis could not identify any statistically significant biological functions related to these modifications. Overall the analysis of ATAC-seq datasets ruled out a putative model where HMGB1 may regulate hepatic lipid metabolism through chromatin packaging. 
Next we sought to determine, using chromatin immuno-precipitation combined with highthroughput sequencing (ChIP-sequencing), whether HMGB1 might exert its activity on gene transcription directly through its abilities to bind DNA. We first set up a reliable and robust ChIP protocol on cells in vitro, as HMGB1 ChIPing might be challenging (26) (fig. S8A-C). Then, using frozen liver samples, we examined HMGB1 binding genome-wide in HMGB ${ }^{\mathrm{fl} / \mathrm{fl}}$ under CD, HFD, and after F/R (Fig.6 and fig. S8-S9). Of note, HMGB1 ChIP-seq was also performed on $\mathrm{HMGB}^{\Delta \mathrm{Hep}}$ livers and these datasets were used as negative control to determine non-specific signals. These background peaks were subtracted in libraries from HMGB1 ${ }^{\mathrm{fl} / \mathrm{fl}}$ livers (fig. S8D-F). Under CD feeding condition, 201250 peaks were detected on the whole genome that were predominantly located in promoters $(18.5 \%)$, introns $(29.3 \%)$ and intergenic regions (32.6\%) (fig. S9A). Interestingly only 155854 and 32006 peaks were detected under the F/R or HFD conditions, respectively, suggesting a significant remodeling of the HMGB1 binding pattern during metabolic stress, even though the qualitative binding remains nearly the same (fig. S9A). The PCA plot of Figure 6A demonstrates significant global differences in HMGB1 DNA occupancy between CD versus F/R and HFD. Venn diagram confirmed this trend with only a few peaks (8859) detected in common in the three conditions (Fig. 6B). The genome browser view of chromosome 3,12 and 14 exemplified the drastic repositioning of HMGB1 upon nutritional stress (Fig. 6C). Along the same lines of observation, partitioning of HMGB1-bound sites by distance to TSSs confirmed the severe change in DNA occupancy of HMGB1. Importantly, the results suggested that most HMGB1 sites located around the TSSs (+/- 3000 bp) under CD feeding were not used under the F/R or HFD conditions (Fig. 6D). Enrichment analysis based on peaks differentially called in CD vs HFD feeding (Fig. 6E) and $\mathrm{CD}$ vs F/R (Fig. 6F) revealed that among several biological functions (GO categories), two are remarkably related to lipid metabolism as the "integration of energy metabolism" and "phospholipid metabolism" (Fig. 6E-F). In these two GO categories, 134 genes displayed a very high occupation rate upon CD compared to F/R and HFD and nearly $90 \%$ of these genes displayed a lower occupancy of HMGB1 in both challenges when compared to CD. These results suggest a common mechanism of regulation in F/R and HFD (Fig. 6G, full list in Table S1 and Table S2). To gain insight into the gene expression program regulated by HMGB1, we performed a motif identification analysis on 134 genes unveiled by the enrichment analysis. The oPOSSUM-3 motif tool revealed the binding motifs of the transcription factors of LXR, identifying this nuclear receptor among the top regulators (Fig. 6H). To functionally test whether the HMGB1 occupancy rate would have an incidence on the level of gene expression, we went back to the microarray data to measure the expression of the 134 genes identified in the 
enrichment analysis performed above. Out of the 134 genes, 70 and 78 are up-regulated in HFD and $\mathrm{F} / \mathrm{R}$, respectively, in livers from $H M G B 1^{\Delta \mathrm{Hep}}$ mice compared to $H M G B 1^{\mathrm{fl} / \mathrm{fl}}$ mice (Fig. 6I-J) providing evidence for a negative correlation between the HMGB1 DNA occupation and the expression of metabolic related-genes identified in the ChIPseq. These data demonstrate that HMGB1 may play a suppressive action on LXR $\alpha$ activity and, consequently, on the level of expression of its target genes.

Taken together, our data are in support of a model whereby at basal state (CD), HMGB1 binds to chromatin loci to modulate the transcription of a number of genes controlled by LXR $\alpha$ which are particularly involved in energy metabolism and lipogenesis.

\section{In vitro, HMGB1 exerts a repressive action on $L X R \alpha$.}

Since HMGB1 modulates chromatin structure and, therefore, regulates transcription factor activity, we examined whether HMGB1 could inhibit $\operatorname{LXR} \alpha$ transcriptional activation in cultured cells transfected with luciferase reporter genes harboring LXR response elements (LXRE). Expression of HMGB1 dramatically decreased LXR $\alpha$ transcriptional activity already at basal state but also after pharmacological activation by synthetic LXR (T093911) or RXR (LG268) agonists (Fig. 7A). Next, we tested whether HMGB1 directly interacts with LXR $\alpha$ in vitro co-immunoprecipitation assays (Fig. 7B) but no interaction could be detected between a flagged Myc-HMGB1 and HA-LXR $\alpha$ (Fig. 7B). These in vitro assays help to firmly establish that HMGB1 is capable of potently repress $\mathrm{LXR} \alpha$ activity at basal state but also upon pharmacological activation, but without any direct physical interaction. Therefore, we tested whether HMGB1 mediated-inhibition of LXR activity may occur through suppressing LXR interaction with the DNA encoding LXR target genes. The ChIP sequencing data suggested that the localization of HMGB1 at specific gene loci correlated with its repressive role of LXR target genes such as Acly or Fasn. These two loci were significantly enriched in CD (green tracks) compared to HFD (purple tracks) and F/R (red tracks) (Fig. 7C-D). Interestingly, HMGB1 bound across the whole loci (Fig. 7C-D) and the promoters of the two HMGB1 repressed genes, Acly or Fasn displayed a heterogeneous HMGB1 occupation patterns (fig. S9B-C), with Acly promoter displaying a high occupation rate in the TSS as opposed to Fasn promoter (fig. S9B-C). This suggests that HMGB1 is not exerting its repressive effect only through TSS occupation. This prompted us to extend the analysis to a series of key genes involved in lipogenesis by performing RT-qPCR experiments on liver samples from adult $\mathrm{HMGB} 1^{\mathrm{fl} / \mathrm{fl}}$ and $\mathrm{HMGB} 1^{\Delta \mathrm{Hep}}$ mice fed with $\mathrm{CD}$, a condition under which HMGB1 repression was strong. The results showed a consistent up-regulation in the expression level of key 
lipogenic genes when HMGB1 was lacking in livers of $\mathrm{HMGB}^{\Delta \mathrm{Hep}}$ mice. The expression of direct LXR $\alpha$ target genes such as Srebf1, Scd-1, Abcg-5 or Abcg-8 and indirect target genes such as Cd36, Cidec, Pnpla3 or Fasn (Fig. 7E) was increased in the liver of these mice compared to their floxed littermates. To establish a causal link between the nuclear presence of HMGB1 and the mRNA expression level of the above-mentioned genes, we deleted HMGB1 selectively in hepatocytes using the hepatocyte-specific promoter of the thyroxine-binding globulin (TBG) gene to express the Cre-recombinase via an AAV8-vector (AAV8-TBG-Cre) in adult $\mathrm{HMGB} 1^{\mathrm{fl} / \mathrm{fl}}$ mice. This strategy was validated by the lower levels of HMGB1 mRNA and protein levels detected in the liver of AAV8-TBG-Cre expressing mice compared to the control group (fig. S10A-B). Remarkably seven days post viral infection with the recombinant virus, the reduced $H m g b 1$ expression resulted in up-regulation of a vast majority of LXR $\alpha$ responsive genes, similarly to what is seen in liver of mice with a constitutive Hmgbl deletion in hepatocytes (Fig. 7F) This result supports a causal and repressive role for HMGB1 on the level of expression of this subset of genes. Overall, these findings support that HMGB1 is repressing LXR $\alpha$ transcriptional activity, which is not mediated by a direct physical interaction with the receptor but rather through a complex DNA occupation across the LXR $\alpha$ responsive gene loci.

\section{Discussion}

Lipogenesis is a fundamental function of the liver to regulate and buffer the amount of circulating lipids, which could present a risk of cellular toxicity in the long run, for numerous tissues (35). Hepatic lipogenesis is therefore tightly regulated by a large number of factors, including TFs and nuclear proteins that together manage positive and repressive actions on gene transcription. These regulatory processes and their interplay are complex and only partly understood and have high relevance due to the high world-wide prevalence of NAFLD (1). Herein, we unraveled a new mechanism regulating liver lipogenesis involving the nuclear factor HMGB1. Using both constitutive and induced knockouts of Hmgbl gene selectively in hepatocytes, we demonstrated that HMGB1, acting in the nucleus, exerts a potent repressive effect on LXR $\alpha$ activity and hepatic lipogenesis during metabolic stresses, such as F/R or HFD feeding, suggesting a protective role on the development of NAFLD.

The nuclear role of HMGB1 might be more complex than initially envisioned and may depend on cell type, nature of environmental signals, and the pathophysiological context. In the context of metabolic stress, we demonstrate in vitro, using primary culture of hepatocyte, that HMGB1 exerts its repressive effect on lipid metabolism in a cell-autonomous manner, thus supporting a model where HMGB1 remains inside the hepatocyte. One can presume that either 
HMGB1 stays in the nucleus and/or translocates in the cytoplasm. Our ChIP-seq data clearly showed that upon the nutritional challenges we have applied, HMGB1 leaves the chromatin, exemplified by reduced binding affinity to DNA and loss of TSS occupancy, triggering a number of changes in gene transcription. Other studies have described a similar impairment of DNA affinity by HMGB1 in cells subjected to stress $(26,40)$. In a recent study, it was shown that in senescent cells, HMGB1 leaves the nucleus leading to a significant change in gene expression (mostly up-regulation) and in chromatin topology (26), which is in agreement with our results in hepatocytes. Despite being poorly documented, it has also been described that HMGB1 in the nucleus may both be bound and unbound to DNA, and that even when unbound it may still reside within the nucleus during cell cycle (40). This supports a model where upon stressors or outside signals, HMGB1 may dissociate from DNA but stays in the nucleus. Yet, the precise mechanisms regulating this biological event and the role of unbound HMGB1 within the nucleus remain unknown, and further experiments are required to understand the underlying mechanism. At the same time, the channeling of HMGB1 between nucleus and cytoplasm is determined by a variety of post-translational modifications such as acetylation, methylation or phosphorylation. During inflammatory challenges for example, acetylation has been described to regulate the accessibility of the HMGB1 nuclear localization signal to the cargo proteins, thus balancing the protein pool between nucleus and cytoplasm (37). In the context of a metabolic stress, it has been suggested that the histone deacetylase SIRT1, a key metabolic sensor (41), may play a significant role in the acetylation status of HMGB1 and its sub-cellular localization (42).

Our data suggest that in response to micro-environmental signals, HMGB1 may dissociate from the chromatin thus affecting biological functions, including metabolic processes. On CD, we found HMGB1 occupying 134 gene loci belonging to metabolic functions, which have been identified as depending on the activity of LXR $\alpha$. As LXR $\alpha$ is a key lipogenic transcription factor involved in cholesterol metabolism and liver lipogenesis, the derepression of its activity induced by HMGB1 deletion logically translates into liver steatosis $(31,32)$. The molecular mechanism behind the inhibition of the hepatic lipogenesis by HMGB1 is still not entirely clear. The immediate mechanism and the simplest scenario would be a direct or indirect binding of HMGB1 with LXR $\alpha$, even though a direct physical interaction was not seen in our co-immunoprecipitation assay (Fig. 7B). One cannot rule out that using more sensitive techniques, a physical interaction might be found as a physical interactions of HMGB1 with transcription factors have been described, notably sterol regulatory element-binding proteins (SREBPs) and the glucocorticoid receptor (GR) (24, 25). Study of the HMGB1- 
interactome in hepatocytes in vivo might be interesting to explore, albeit technically challenging.

Our ATAC-seq data helped to demonstrate that chromatin compaction was not regulated by HMGB1 under $\mathrm{CD}$ and during the nutritional challenges (fig. S7), suggesting that the HMGB1-mediated repression was likely not mediated through a nucleosomal re-organization. This hypothesis was important to test, as several reports demonstrated a key role of HMGB1 in the nucleosome arrangement remodeling associated to transcription modulation in vitro (22). At least in the in vivo context of liver steatosis, our results support a minor role for HMGB1 in regulating nucleosomal landscapes, which represents a significant layer of epigenetic control of transcription. However, our ChIP-seq data suggested DNA occupancy as a likely mechanism of repression. HMGB1 has a very high level of DNA occupation in the basal state and that it is located equally in the promoter region, $\mathrm{CDS}$ and distal intergenic region. However, upon metabolic stress, HMGB1 appears to leave the chromatin, particularly the TSS regions (Fig. 7C). This suggests that HMGB1 DNA occupancy is correlated with changes in gene transcription, but interestingly, the occupancy rate in the TSS is not necessarily related to the level of repression, as shown by two equally-repressed genes (Acly and Fasn) with heterogeneous TSS occupation (fig. S9B-C). Hence, occupancy appears to be an important factor, but likely not the only one. Of note, our data using inducible Hmgbl deletion via AAV8TBG-Cre show that the absence of HMGB1 consistently leads to the up-regulation of genes involved in hepatic lipogenesis, suggesting a causal relationship between HMGB1 and gene expression (Fig. 7E-F). These results are corroborated by a study of Sofiadis et al, depicting a map of HMGB1 binding genome-wide in senescent cells using a combination of RNA-seq, ChIP-seq and Hi-C (chromatin conformation capture). Interestingly, in primary cells at senescent state, HMGB1 leaves the chromatin, triggering profound changes in chromatin dynamics and gene transcription, in a similar fashion as seen by us. Additionally, Hi-C data demonstrated that HMGB1 binds to TAD (Topology Associated Domain) boundaries, known to regulate chromatin topology and consequently gene expression. In addition to this paper, a recent study has also evoked an RNA-binding property as a another functional layer for HMGB1 to regulate gene expression $(26,43)$. Therefore, 3-D conformation and RNA binding clearly represent additional mechanisms by which HMGB1 could mediate its repressive effect on LXR $\alpha$, which is therefore worthwhile to further investigate in the context of liver steatosis.

Overall our study helped to uncover HMGB1-mediated $\operatorname{LXR} \alpha$ repression as new mechanism modulating liver lipogenesis during metabolic stress. Boosting these functions of 
HMGB1 may constitute a new therapeutic approach to counteract the deleterious effect of enhanced LXR $\alpha$ activity in patients with NAFLD.

\section{Materials and Methods}

\section{Experimental Design}

This study aimed to decipher the precise role of the nuclear factor HMGB1 in hepatocytes during metabolic stress. For this, a cell specific knockout mice model where Hmgbl gene is deleted specifically in hepatocytes $\left(\mathrm{HMGB} 1^{\Delta \mathrm{Hep}}\right)$ and its control counterpart $\left(\mathrm{HMGB} 1^{\mathrm{fl} / \mathrm{fl}}\right)$ were subjected to nutritional stress such as high fat diet and fasting/refeeding. A combination of OMICS studies has been employed to nail down the potential mechanism behind HMGB1 repressive effect on hepatic lipogenesis such as microarray, ATAC-seq or ChIP-seq. All studies identified lipid metabolism as a key function and transcription factor LXR $\alpha$ as a key piece that might be repressed by HMGB1. In vivo studies using adenovirus-mediated shRNA expression targeting LXR $\alpha$ were employed to functionally test the interdependence of HMGB1 and LXR $\alpha$. In vitro assays were used to measure how HMGB1 could regulate the transcriptional activation using specific responsive elements (RE)-containing luciferase reporter. For in vivo studies, adult age-matched Cre +/- carrying Hmgb1 floxed gene called HMGB1 ${ }^{\Delta \text { Hep }}$ mice and their control Cre -/- carrying Hmgb1 floxed gene named HMGB1 ${ }^{\mathrm{fl} / \mathrm{fl}}$ littermates were co-housed to reduce variability. Animal numbers for each study type were determined by the investigators on the basis of data from previous similar experiments or from pilot studies. For OMICS studies, displayed animals were chosen as representative from the whole cohort: (i) for the microarray 4 animals per genotype/per challenge, (ii) 2 animals per genotype/per challenge for the Chip-seq and (ii) 2 animals per genotype/per challenge for the ATAC-seq have been analyzed. For neutral lipid analysis and histology experiments, sample identities were not known in most cases and were randomized. For in vitro studies, at least three biological replicates were used in three separate experiments.

\section{Mouse Phenotyping}

Breeding and experimental procedures were performed in accordance with institutional guidelines for animal research and were approved by the Animal Care and Use Ethics Committee US006 CREFRE - CEEA-122 (protocol 17/1048/03/20). Animals were housed in temperature and humidity controlled facilities under a 12 hour-light period with free access to food and water. All animals were aged between 2 to 3 months at the beginning of the experimentations. Hepatocyte-specific deletion of $\mathrm{Hmgbl}$ gene noted HMGB1 ${ }^{\Delta \mathrm{Hep}}$ were 
557 generated crossing Alb-CRE ${ }^{+/}$(Jackson Laboratory, Ban Harbor, ME, USA) with Hmgb1

558 floxed mice noted HMGB1 ${ }^{\mathrm{fl} / \mathrm{fl}}$ (a generous gift from Dr. Robert F. Schwabe, Columbia

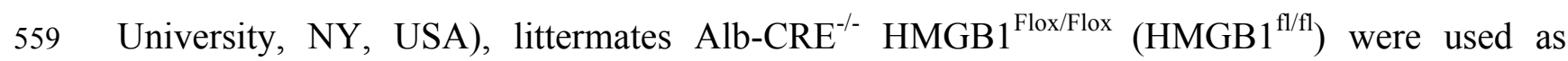
560 control. Hepatocyte-specific deletion of Ppary gene noted PPAR $\gamma^{\Delta \mathrm{Hep}}$ were generated crossing 561 Alb-CRE $^{+-}$(Jackson Laboratory, Ban Harbor, ME, USA) with Ppary floxed mice noted $562 \operatorname{PPAR} \gamma^{\mathrm{fl} / \mathrm{fl}}$ (a generous gift from Pr. W.A Wahli, University of Lausanne, Switzerland), 563 littermates Alb-CRE ${ }^{-/-} \operatorname{PPAR} \gamma^{\text {Flox/Flox }}\left(\operatorname{PPAR} \gamma^{\mathrm{fl} / \mathrm{fl}}\right)$ were used as control. At the time of sacrifice, 564 tissues and organs were dissected, weighted and directly snap frozen in liquid nitrogen and 565 stored at $-80^{\circ} \mathrm{C}$.

\section{Genotyping}

DNA extraction and PCR were performed using Kapa mouse genotyping kit (Kapa Biosystems, Wilmington, MA, USA) according to the manufacturer protocol. PCR reactions were performed using following primers: Alb-CRE: 5'-ACCGGTCGATCGAAACGAGTGATGAG-3 (forward) and 5'-AGTGCGTTCGAACGCTAGAGC-3' (reverse), LoxP1 5 -

TAAGAGCTGGGTAAACTTTAGGTG-3'

(forward)

and

5'-

GAAACAGACAAGCTTCAAACTGCT-3'

(reverse),

LoxP2

5'-

TGACAGGATACCCAGTGTTAGGGG-3'

(forward)

and

5 '-

CCAGAGTTTAATCCACAGAAGAAA-3’ (reverse).

\section{Interventional experiments}

-For diet induced-obesity experiments, mice were fed with a normal chow diet (CD, Research Diets, New Brunswick, NJ, USA) or a high fat diet (HFD60\%, Research Diets, New Brunswick, NJ, USA) for 12 or 24 weeks. To induce liver steatosis, mice were subjected to HFD60\% with $30 \%$ fructose (Sigma-Aldrich, Saint Louis, MO, USA), dissolved in the drinking water or choline deficient diet supplemented with 60\% fat (CD-HFD60\%, Research Diets, New Brunswick, NJ, USA). For the fasting-refeeding, mice under normal chow diet (CD) were starved 6 hours from Zeitgeber 14 (ZT14) and refeed for 8 hours with the CD and 20\% glucose (Sigma-Aldrich, Saint Louis, MO, USA) in the drinking water.

-Body composition was assessed using the EchoMRI (Echo Medical Systems, Houston, TX, USA).

-Indirect calorimetry was performed after $24 \mathrm{~h}$ of acclimatization in individual cages. Oxygen consumption, carbon dioxide production, and food and water intake were measured

(Phenomaster; TSE Systems, Bad Homburg v.d.H, Germany) in individual mice at 15-min 
intervals during a $24-\mathrm{h}$ period at constant temperature $\left(22^{\circ} \mathrm{C}\right)$. The respiratory exchange ratio $\left([\mathrm{RER}]=V \mathrm{CO}_{2} / V \mathrm{O}_{2}\right)$ was measured. The glucose oxidation $\left(\right.$ in $\mathrm{g} / \mathrm{min} / \mathrm{kg}^{0.75}=\left[\left(4.545 \times V \mathrm{co}_{2}\right)-\right.$ $\left.\left.\left(3.205 \times V \mathrm{o}_{2}\right)\right] / 1000\right)$ and lipid oxidation $\left(\right.$ in $\left.\mathrm{g} / \mathrm{min} / \mathrm{kg}^{0.75}=\left[1.672 \times\left(V_{\mathrm{o}_{2}}-V \mathrm{co}_{2}\right)\right] / 1000\right)$ were calculated. Ambulatory activities of the mice were monitored by infrared photocell beam interruption (Sedacom; Panlab-Bioseb).

-For Hmgbl gene deletion at adult age, HMGB1 ${ }^{\mathrm{fl} / \mathrm{fl}}$ male mice at 8 weeks of age were injected intravenously (i.v) with $10^{11}$ genomic copies per mouse with adeno-associated-virus (AAV8) containing a liver-specific promoter, thyroxine-binding globulin (TBG) promoter driving either GFP or Cre recombinase (Penn Vector Core, University of Pennsylvania, PA, USA) to generate control mice noted AAV-GFP or liver specific HMGB1 knockout noted AAV-CRE. 7 days after injections, animals were euthanized.

- To knockdown LXR, adult male HMGB1 $1^{\text {fl/fl }}$ and $H M G B 1^{\Delta \text { Hep }}$ mice (8-12 week-old) were injected i.v with an adenovirus expressing an shRNA targeting LXR $\alpha$ (kindly provided by Dr Catherine Postic, Cochin Institute, Paris, France). For both adenovirus protocols, $10^{13}$ adenoviral infectious particles were diluted in $0.9 \% \mathrm{NaCl}$ and administered in a total volume of $100 \mu \mathrm{l}$ per animal. 7-10 days after injection, control (scramble RNA noted $\operatorname{sh} S C R$ ) and $\operatorname{sh} L X R \alpha$ expressing mice were subjected to fasting/refeeding challenges as described previously. To study HFD-induced liver steatosis, mice were first subjected to a 4 week-HFD60\%, then injected with $\operatorname{sh} S C R$ and $\operatorname{sh} L X R \alpha$, and mice were euthanized 7-10 post injections.

-For Insulin acute injection, CD or HFD $60 \%$ fed $\mathrm{HMGB} 1^{\mathrm{fl} / \mathrm{fl}}$ and $\mathrm{HMGB} 1^{\Delta \mathrm{Hep}}$ mice were fasted for 16 hours and then injected i.p (intra-peritoneal) with $0,75 \mathrm{U} / \mathrm{kg}$ of human insulin and mice were sacrificed 15 minutes later.

-For LXR in vivo activation, synthetic agonist T0901317 (30mg/kg, Bertin Bioreagent, Montigny le Bretonneux, France) was administered orally by four consecutive daily gavages on 8-week-old $\mathrm{HMGB} 1^{\mathrm{fl} / \mathrm{fl}}$ and $\mathrm{HMGB} 1^{\Delta \mathrm{Hep}}$ adult male mice. Mice were starved one hour before the fourth gavage, and maintained starved for 5 more hours before euthanasia.

- For hepatic VLDL-triacylglycerol production assay, 8-week-old HMGB1 ${ }^{\mathrm{fl} / \mathrm{fl}}$ and HMGB1 ${ }^{\Delta \mathrm{Hep}}$ adult male mice fasted overnight received an intravenous injection of $10 \%$ tyloxapol (500 mg/kg) (Sigma-Aldrich, T8761, Saint Louis, MO, USA). Blood was collected from the tail vein at $0,1,2,3$ and 4 hours for triglyceride assays.

\section{Glucose/Insulin/pyruvate tolerance test}

-Glucose (GTT), Insulin (ITT) and pyruvate (PTT) tolerance tests were performed under chow diet or after 12 weeks of HFD after an overnight fast. Glucose (Sigma, G8270, Saint Louis, 
MO, USA) was orally administered at $1.5 \mathrm{~g} / \mathrm{kg}$ dose, Insulin was injected i.p at $0.75 \mathrm{U} / \mathrm{kg}$ and pyruvate (Sigma, P2256, Saint Louis, MO, USA) was administrated by i.p. injection at $1.5 \mathrm{~g} / \mathrm{kg}$. For all tolerance tests the glycaemia evolution was then monitored at the tail vein using AccuCheck glucometer (Roche). Plasma insulin (Mercodia, Upasal, Sweden) was determined by ELISA in the fasted state or at indicated times.

\section{Primary Hepatocyte Isolation}

Mouse hepatocytes were isolated as previously described via 2-step collagenase perfusion as described by Fortier et al (44). Hepatocytes were allowed to attach for 90 minutes on collagencoated plates in RPMI containing 10\% FBS (Gibco), followed by overnight starvation in serumfree medium before experiments (Lipogenesis and $\beta$-oxidation assay).

\section{Lipogenesis assays}

-For in vitro measurement, one day after isolation, primary hepatocytes were serum-starved for 3 hours and incubated for 3 -hour with $\left[1-{ }^{14} \mathrm{C}\right]$ acetate $(1 \mu \mathrm{Ci} / \mathrm{ml}$; Perkin Elmer, Boston, MA) and $5.5 \mathrm{mM}$ of non-labeled (cold) glucose in DMEM medium. At the end of incubation, cells were washed twice with cold PBS $1 \mathrm{X}$ and harvested into $0.25 \mathrm{ml}$ of $0.1 \%$ SDS for subsequent protein measurement and total lipid extraction with $1 \mathrm{ml}$ of chloroform/methanol $(2 \mathrm{v} / 1 \mathrm{v})$. Lipid extracts were washed with $70 \%$ ethanol, and then dissolved into chloroform/methanol $(2 \mathrm{v} / 1 \mathrm{v})$. Radioactivity was measured on a multipurpose scintillation counter (LS 6500; Beckman Coulter). All assays were performed in duplicates, and data normalized to cell protein content. -For in vivo measurement of lipogenesis activity, animals were fasted for 6 hours at ZT14 and received an i.p. bolus of $2 \mathrm{mg} / \mathrm{g}$ glucose containing $0.4 \mu \mathrm{Ci} / \mathrm{g}$ of [3-3H]-D-glucose (PerkinElmer, NET331C, Waltham, MA, USA). After 1 hour, liver, epididymal, subcutaneous and brown adipose tissues were collected and snap-frozen in liquid nitrogen.

- For palmitate oxidation assay: Cells were preincubated for 3 hours with ${ }^{14}$ Cpalmitate $(1 \mathrm{uCi} / \mathrm{mL}$; Perkin Elmer, Boston MA) and non labeled (cold) palmitate. Palmitate was coupled to a fatty acid-free BSA in a molar ratio of 5:1. Following incubation, ${ }^{14} \mathrm{CO}_{2}$ and ${ }^{14} \mathrm{C}$-ASM were measured as previously described (45). Briefly, assayed medium was transferred into a custommade Teflon 48-well trapping plate. The plate was clamped and sealed, and perchloric acid was injected through the perforations in the lid into the medium, which drives $\mathrm{CO}_{2}$ through the tunnel into an adjacent well, where it was trapped in $1 \mathrm{~N} \mathrm{NaOH}$. Following trapping, the media was spun twice and ${ }^{14} \mathrm{C}$-ASM measured by scintillation counting. Aliquots of $\mathrm{NaOH}$ and medium were transferred into scintillation vials, and radioactivity was measured on a 
multipurpose scintillation counter (LS 6500; Beckham Coulter). All assays were performed in triplicates, and data were normalized to protein content.

\section{Liver neutral lipid analysis}

Hepatic lipids were extracted by the "Folch" procedure before being quantified using mass spectrometry. Briefly, 50mg of liver were homogenized in $1 \mathrm{~mL}$ water:methanol $(1: 2 \mathrm{v} / \mathrm{v}), 5$ mM EGTA. Lipids are then extracted using a methanol: chloroform: water $(2.5: 2.5: 1.7 \mathrm{v} / \mathrm{v})$ mix. After a solid phase extraction, purification and desiccation, all lipids are eluted in ethylacetate and analyzed by a gas chromatography combined with mass spectrometry (GC-MS) (ISQ Thermo).

\section{Microarray Gene Expression Studies}

Gene expression profiles were performed at the GeT-TRiX facility (GénoToul, Génopole Toulouse Midi-Pyrénées) using Agilent Sureprint G3 Mouse GE v2 microarrays (8x60K, design 074809) following the manufacturer's instructions. For each sample, Cyanine-3 (Cy3) labeled cRNA was prepared from $200 \mathrm{ng}$ of total RNA using the One-Color Quick Amp Labeling kit (Agilent) according to the manufacturer's instructions, followed by Agencourt RNAClean XP (Agencourt Bioscience Corporation, Beverly, Massachusetts). Dye incorporation and cRNA yield were checked using Dropsense ${ }^{\text {TM }} 96$ UV/VIS droplet reader (Trinean, Belgium). 600 ng of Cy3-labelled cRNA were hybridized on the microarray slides following the manufacturer's instructions. Immediately after washing, the slides were scanned on Agilent G2505C Microarray Scanner using Agilent Scan Control A.8.5.1 software and fluorescence signal extracted using Agilent Feature Extraction software v10.10.1.1 with default parameters.

\section{Microarray data statistical analysis:}

Microarray data were analyzed using R (46) and Bioconductor packages (47). Raw data (median signal intensity) were filtered, $\log 2$ transformed and normalized using the quantile method (48) with the limma package (49).

A model was fit using the limma lmFit function (49). Pairwise comparisons between biological conditions were applied using specific contrasts. In cases where Agilent has multiple probe sequences for the same gene, the probe with the best p-value was selected. Probes with a pvalue $\leq 0.01$ were considered to be differentially expressed between conditions.

Normalized log intensities were averaged $(\mathrm{n}==4)$ within each group and heatmaps were generated with the ComplexHeatmap package (50). Venn diagrams were generated with the 
Vennerable package (https://github.com/js229/Vennerable). Functional pathway enrichment was performed in $\mathrm{R}$ using the hypergea package's hypergeometric test (https://cran.rproject.org/package=hypergea). GO annotations were obtained using biomaRt (51) and the graphite package (52) was used to obtain pathways from the Reactome database. ChEA (https://doi.org/10.1093/bioinformatics/btq466) was interrogated via the Enrichr website (53) and tabular results were imported into R. Barcharts were constructed using ggplot2 (54). The network of pathways largely shared between F/R and HFD was constructed in R as csv files that were imported into Cytoscape (55).

\section{ChIP-seq}

Briefly, frozen liver biopsies (100-200 mg) harvested from HMGB1 $1^{\mathrm{fl} / \mathrm{fl}}$ and HMGB1 $1^{\Delta \mathrm{Hep}}$ mice under $\mathrm{CD}$, upon $\mathrm{HFD} 60 \%$ or after $\mathrm{F} / \mathrm{R}$, were minced and fixed at room temperature in PBS-1\% formaldehyde (Sigma-Aldrich, 47608, Saint Louis, MO, USA) for 20 minutes. After sonication, chromatin immunoprecipitation was performed using anti-HMGB1 antibody (Abcam, ab18256, Cambridge, UK). Immunoprecipitated DNA was subjected to library preparation and single-end sequencing on a NextSeq 500 at EMBL GeneCore (Heidelberg, Germany).

\section{ATAC-seq}

Flash-frozen liver biopsies were sent to Active Motif to perform the ATAC-seq assay. The tissue was manually dissociated, isolated nuclei were quantified using a hemocytometer, and 100,000 nuclei were tagmented as previously described (56), with some modifications based on (57) using the enzyme and buffer provided in the Nextera Library Prep Kit (Illumina). Tagmented DNA was then purified using the MinElute PCR purification kit (Qiagen), amplified with 10 cycles of PCR, and purified using Agencourt AMPure SPRI beads (Beckman Coulter). Resulting material was quantified using the KAPA Library Quantification Kit for Illumina platforms (KAPA Biosystems), and sequenced with PE42 sequencing on the NextSeq 500 sequencer (Illumina).

\section{ATAC-seq and ChIP-seq data analysis}

ATAC-seq and ChIP-seq reads were first mapped to the mouse genome UCSC build hmm10 using Bowtie2 2.2.8 (58). Aligned reads were then filtered to keep only matched pairs and uniquely mapped reads. Peaks were called with MACS2 2.2.1 (59) algorithm using a mappable genome size of $2.73 \mathrm{e}^{9}$. To process ChIP-seq datasets, MACS2 was run with the "Delta" genotype as a negative control as in this condition the HMGB1 protein expression is reduced by 
$90 \%$ and signal detected in "Delta" libraries, defined as background noise, was substracted from the "Flox" libraries. ATAC-seq datasets were processed without a control file and with the -nomodel option. Called peaks that were on the ENCODE blacklist of known false ChIP-seq peaks were removed. Signal maps and peak locations were used as input to the statistical analysis performed with the $\mathrm{R}$ package ChIPseeker (60). DESeq2 (61) was used to identify differential binding sites and differential open chromatin profiles. Motifs and GO enrichment analysis were respectively performed using JASPAR (62) and the R package ReactomePA (63).

Histology: Tissue samples were fixed in 10\% formalin (Sigma-Aldrich, HT501128, Saint Louis, MO, USA) for 24 hours, then incubated at $4{ }^{\circ} \mathrm{C}$ in $70 \%$ ethanol before being paraffinembedded or in $30 \%$ sucrose before being cryo-embedded with Tissue-Tek OCT (Sakura FineTek Europe, Alphen aan den Rijn, The Netherlands). Paraffin embedded livers were sliced at $5 \mu \mathrm{m}$. For Periodic Acid Schiff reaction, sections are incubated in $0.5 \%$ periodic acid in water for 5 minutes then transferred to Schiff reagent (Sigma-Aldrich, 3952016, Saint Louis, MO, USA) for 15 minutes. Sections were counterstained with Mayer's hematoxylin (Sigma-Aldrich, MHS16, Saint Louis, MO, USA) before mounting. Liver-cryo sections were post-fixed with 10\% formalin 15 minutes prior staining with Oil-red-O (Sigma-Aldrich, MHS16, Saint Louis, MO, USA)(60\% solution in isopropanol-Sigma-Aldrich, 33539, Saint Louis, MO, USA). After counter-staining with hematoxylin, slides are mounted with aqueous mounting media. Stained slides were scanned using a Nanozoomer scanner (Hamamatsu Photonics, Hamamatsu City, Japan). Images quantification was performed using Image J freeware (NIH, USA).

\section{Western blotting}

Tissues were homogenized in RIPA buffer (TRIS 20 mM, NaCl 150 mM, EDTA 1 mM, EGTA $1 \mathrm{mM}$, TRITON X100 1\%, Tetra-Sodium Pyrophosphate $2.5 \mathrm{mM}$, B-Glycerophosphate $1 \mathrm{mM}$, Sodium orthovanadate $1 \mathrm{mM}$ ) containing protease and phosphatase inhibitors (Sigma-Aldrich, St. Louis, MO, USA) using Precellys sample lyzer (Bertin Technologies, Montigny le Bretonneux, France). Western blots were performed using standard procedures using antibodies against HMGB1 (1:1000, ab18256, Abcam, Cambridge, UK), Phospho-AKT S473 (1:1000, CST 4060, Cell Signaling Technology, Danvers, MA, USA), total AKT (1:1000, CST 9272, Cell Signaling Technology, Danvers, MA, USA), HA (1:1000, CST 3724 Cell Signaling Technology, Danvers, MA, USA), Myc-tag (1:1000, CST 2276, Cell Signaling Technology, Danvers, MA, USA) and GAPDH (1: 2000, ab181602, Abcam, Cambridge, UK), was used as a loading control. 


\section{Reporter assay}

For reporter assay, Ad293 cells were cultured in 96 well plates with DMEM containing 10\% FB Essence (Avantor Seradigm, USA) and transfected using Transit-LT1 (Mirus Bio, Madison, WI, USA) with plasmid encoding 4 LXR response elements fused with luciferase, human HA-LXR (HA-hLXR) and RXR. HMGB1 plasmid was purchased from Origene. 24h after transfection, cells media was changed to DMEM containing 2\% charcoal striped and dialyzed media with 0.1 uM of T0901317 and/or 1uM of LG100268 (noted LG268) (Cayman Chemical, USA). After overnight treatment, luciferase activity was assayed using a luciferase assay system (Promega, USA). Bioluminescence was quantified using a luminometer and normalized to $\beta$-Gal activity.

\section{Co-immunoprecipitation}

Ad293 cells were plated in 6 well-plate and transfected as previously described with 1 ug of HA-hLXR and/or HMGB1 plasmids. 24h after transfection, cells were treated with $0.1 \mathrm{uM}$ of T0901317 overnight. Cells were lysed in IP buffer $(20 \mathrm{mM}$ Tris-HCl pH8, 100mM NaCl, $0.1 \%$ NP40, 10\% glycerol, 2uM PMSF and 1mM DTT) supplemented with antiprotease and antiphosphatase cocktails.

IP was performed using HA-conjugated beads (Sigma) for $2 \mathrm{~h}$ at $4^{\circ} \mathrm{C}$, following wash step, beads were resuspended in $2 \mathrm{X}$ Laemmli buffer and western blot was performed as previously described.

\section{Gene expression}

RNA were extracted using GenJET RNA purification kit (ThermoScientific, Waltham, MA, USA) and DNAse treatment (Qiagen, Hilden, Germany). After dosage with Xpose (Trinean, Gentbrugge, Belgium) reverse transcription was performed using High Capacity cDNA reverse transcription kit (Applied Biosystem, Foster City, CA, USA) according to the manufacturer protocol. Real-Time -qPCR was performed with indicated primer pairs gene expression is normalized using $36 b 4$ reference gene expression. Primer sequences are available in Table S3.

\section{Microfluidic qPCR}

Expression analyses of lipogenesis related-genes (Table S3) were performed by quantitative PCR with Fluidigm Biomark ${ }^{\circledR}$ technology (Genome \& Transcriptome GenoToul Platform). First-strand cDNA templates were pre-amplified with Preamp Master Mix (Fluidigm) and reactions were achieved in a Fluidigm Biomark ${ }^{\circledR}$ BMK-M-96.96 plate according to the manufacturer's recommendations. Relative gene expression values were determined using the 
$7952^{-\triangle \Delta \mathrm{CT}}$ method. The expression analyses data are an average of seven individuals for $\mathrm{HMGB} 1^{\mathrm{fl} / \mathrm{fl}}$ mice and 10 individuals for HMGB1 ${ }^{\Delta \mathrm{Hep}}$ mice. As described before, the $36 B 4$ gene expression levels were used for data standardization.

\section{Plasma analysis}

Whole blood is drawn out from the inferior vena cava after euthansia, and plasma is prepared after centrifugation (5 minutes; $4{ }^{\circ} \mathrm{C} ; 8000 \mathrm{rpm}$ ). Circulating AST (ASpartate aminoTransferase) and ALT (ALanine aminoTransferase) levels were determined in plasma by the Phénotypage-CREFRE facility using a Pentra400 biochemical analyzer (HORIBA Medical, Kyoto, Japan). HMGB1 circulating levels were assessed by ELISA (ST51011, IBL International, Hamburg, Germany) on $10 \mathrm{uL}$ of plasma, according to the manufacturer guidelines.

\section{Statistics}

Analyses are performed using GraphPad Prism 7 (GraphPad Software, La Jolla, CA, USA). Potential outliers were identified using ROUT algorithm (GraphPad Software) and removed from analysis. All data are expressed as mean \pm SEM, except otherwise indicated. Statistical significance was determined by Mann \& Withney, one-way ANOVA or two-way ANOVA followed by a Tuckey post-hoc test. P values $<0.05$ were considered significant $\left(* p<0.05 ; *^{*} p\right.$ $<0.01 ; * * * \mathrm{p}<0.001 ; * * * * \mathrm{p}<0,0001)$.

\section{H2: Supplementary Materials}

Fig. S1. Metabolic explorations of Hepatocyte specific Hmgbl deleted mice subjected to chowdiet.

Fig. S2. Metabolic explorations of Hepatocyte specific Hmgbl deleted mice subjected to highfat diet.

Fig. S3. Hepatocyte specific Hmgb1 deleted mice exhibit a severe liver steatosis upon various diets.

Fig. S4. Hierarchical clustering and color heatmap of differentially expressed gene comparing livers of $\mathrm{HMGB}^{\mathrm{fl} / \mathrm{fl}}$ and $\mathrm{HMGB} 1^{\Delta \mathrm{Hep}}$ mice.

Fig. S5. Hepatocyte specific Ppary deletion does not modify liver steatosis in mice after F/R challenge.

Fig. S6. Hepatocyte specific Hmgbl deleted mice exhibit a severe liver steatosis under metabolic stressors that is restored by knocking-down $\mathrm{LXR} \alpha$ in vivo.

Fig. S7. Hepatocyte specific Hmgbl deletion does not remodel chromatin.

Fig. S8. Validation of HMGB1 ChIP in vitro and in vivo.

Fig. S9. Genomic and around TSS $(-1 \mathrm{~kb} /+1 \mathrm{~kb})$ region distribution of HMGB1.

Fig. S10. Successful in vivo knockdown of Hmgbl gene and protein using AAV-TBG-Cre. 
Table S1. List of genes highly occupied by HMGB1 in Chow Diet compare to HFD

Table S2. List of genes highly occupied by HMGB1 in Chow Diet compare to F/R

Table S3. Primers for Real time qPCR

838

839

840

841

842

843

844

\section{References and Notes}

1. Z. Younossi, Q. M. Anstee, M. Marietti, T. Hardy, L. Henry, M. Eslam, J. George, E. Bugianesi, Global burden of NAFLD and NASH: Trends, predictions, risk factors and prevention. Nat. Rev. Gastroenterol. Hepatol. 15 (2018), pp. 11-20.

2. Q. M. Anstee, H. L. Reeves, E. Kotsiliti, O. Govaere, M. Heikenwalder, From NASH to HCC: current concepts and future challenges. Nat. Rev. Gastroenterol. Hepatol. 16 (2019), pp. 411-428.

3. Y. Wang, J. Viscarra, S.-J. Kim, H. S. Sul, Transcriptional regulation of hepatic lipogenesis. Nat. Rev. Mol. Cell Biol. 16, 678-89 (2015).

4. B. Wang, $\mathrm{P}$. Tontonoz, Liver $\mathrm{X}$ receptors in lipid signalling and membrane homeostasis. Nat. Rev. Endocrinol. 14 (2018), pp. 452-463.

5. S. Ducheix, A. Montagner, A. Polizzi, F. Lasserre, A. Marmugi, J. Bertrand-Michel, N. Podechard, T. Al Saati, M. Chétiveaux, S. Baron, J. Boué, G. Dietrich, L. Mselli-Lakhal, P. Costet, J. M. A. Lobaccaro, T. Pineau, V. Theodorou, C. Postic, P. G. P. Martin, H. Guillou, Essential fatty acids deficiency promotes lipogenic gene expression and hepatic steatosis through the liver X receptor. J. Hepatol. 58, 984-992 (2013).

6. D. J. Peet, S. D. Turley, W. Ma, B. A. Janowski, J. M. A. Lobaccaro, R. E. Hammer, D. J. Mangelsdorf, Cholesterol and bile acid metabolism are impaired in mice lacking the nuclear oxysterol receptor LXR $\alpha$. Cell. 93, 693-704 (1998).

7. S. Ducheix, A. Montagner, A. Polizzi, F. Lasserre, M. Régnier, A. Marmugi, F. Benhamed, J. Bertrand-Michel, L. Mselli-Lakhal, N. Loiseau, P. G. Martin, J.-M. Lobaccaro, L. Ferrier, C. Postic, H. Guillou, Dietary oleic acid regulates hepatic lipogenesis through a liver $\mathrm{X}$ receptor-dependent signaling. PLoS One. 12, e0181393 (2017).

8. Y. Wang, J. Viscarra, S. J. Kim, H. S. Sul, Transcriptional regulation of hepatic lipogenesis. Nat. Rev. Mol. Cell Biol. 16 (2015), pp. 678-689.

9. J. Viscarra, H. S. Sul, Epigenetic regulation of hepatic lipogenesis: Role in hepatosteatosis and diabetes. Diabetes. 69, 525-531 (2020).

10. J. H. Kim, D. Y. Jung, H. R. Kim, M. H. Jung, Histone h3k9 demethylase jmjd2b plays a role in lxra-dependent lipogenesis. Int. J. Mol. Sci. 21, 1-17 (2020).

11. S. W. Beaven, A. Matveyenko, K. Wroblewski, L. Chao, D. Wilpitz, T. W. Hsu, J. Lentz, B. Drew, A. L. Hevener, P. Tontonoz, Reciprocal regulation of hepatic and adipose lipogenesis by liver X receptors in obesity and insulin resistance. Cell Metab. 18, 106117 (2013).

12. N. Y. Kalaany, K. C. Gauthier, A. M. Zavacki, P. P. A. Mammen, T. Kitazume, J. A. Peterson, J. D. Horton, D. J. Garry, A. C. Bianco, D. J. Mangelsdorf, LXRs regulate the balance between fat storage and oxidation. Cell Metab. 1, 231-244 (2005).

13. J. J. Repa, G. Liang, J. Ou, Y. Bashmakov, J. M. A. Lobaccaro, I. Shimomura, B. Shan, M. S. Brown, J. L. Goldstein, D. J. Mangelsdorf, Regulation of mouse sterol regulatory element-binding protein-1c gene (SREBP-1c) by oxysterol receptors, LXR $\alpha$ and LXR $\beta$. Genes Dev. 14, 2819-2830 (2000).

14. B. L. Heckmann, X. Zhang, A. M. Saarinen, G. Schoiswohl, E. E. Kershaw, R. Zechner, 
J. Liu, Liver $\mathrm{X}$ receptor $\alpha$ mediates hepatic triglyceride accumulation through upregulation of G0/G1 Switch Gene 2 expression. JCI Insight. 2 (2017), doi:10.1172/jci.insight. 88735 .

15. T. G. Kirchgessner, P. Sleph, J. Ostrowski, J. Lupisella, C. S. Ryan, X. Liu, G. Fernando, D. Grimm, P. Shipkova, R. Zhang, R. Garcia, J. Zhu, A. He, H. Malone, R. Martin, K. Behnia, Z. Wang, Y. C. Barrett, R. J. Garmise, L. Yuan, J. Zhang, M. D. Gandhi, P. Wastall, T. Li, S. Du, L. Salvador, R. Mohan, G. H. Cantor, E. Kick, J. Lee, R. J. A. Frost, Beneficial and Adverse Effects of an LXR Agonist on Human Lipid and Lipoprotein Metabolism and Circulating Neutrophils. Cell Metab. 24, 223-233 (2016).

16. H. Yang, H. Wang, U. Andersson, Targeting Inflammation Driven by HMGB1. Front. Immunol. 11 (2020), , doi:10.3389/fimmu.2020.00484.

17. G. H. Goodwin, C. Sanders, E. W. Johns, A New Group of Chromatin-Associated Proteins with a High Content of Acidic and Basic Amino Acids. Eur. J. Biochem. 38, 14 19 (1973).

18. I. PJ, F. JL, B. DL, R. GR, Preferential affinity of high molecular weight high mobility group non-histone chromatin proteins for single-stranded DNA. J. Biol. Chem. 254 (1979).

19. M. Štros, HMGB proteins: Interactions with DNA and chromatin. Biochim. Biophys. Acta - Gene Regul. Mech. 1799 (2010), pp. 101-113.

20. H. Ito, K. Fujita, K. Tagawa, X. Chen, H. Homma, T. Sasabe, J. Shimizu, S. Shimizu, T. Tamura, S. Muramatsu, H. Okazawa, HMGB 1 facilitates repair of mitochondrial DNA damage and extends the lifespan of mutant ataxin-1 knock-in mice . EMBO Mol. Med. 7, 78-101 (2015).

21. M. Watson, K. Stott, H. Fischl, L. Cato, J. O. Thomas, Characterization of the interaction between HMGB1 and H3-a possible means of positioning HMGB1 in chromatin, doi:10.1093/nar/gkt950.

22. B. Celona, A. Weiner, F. Di Felice, F. M. Mancuso, E. Cesarini, R. L. Rossi, L. Gregory, D. Baban, G. Rossetti, P. Grianti, M. Pagani, T. Bonaldi, J. Ragoussis, N. Friedman, G. Camilloni, M. E. Bianchi, A. Agresti, Substantial Histone reduction modulates Genomewide nucleosomal occupancy and global transcriptional output. PLoS Biol. 9 (2011), doi:10.1371/journal.pbio.1001086.

23. T. Imamura, H. Izumi, G. Nagatani, T. Ise, M. Nomoto, Y. Iwamoto, K. Kohno, Interaction with p53 Enhances Binding of Cisplatin-modified DNA by High Mobility Group 1 Protein. J. Biol. Chem. 276, 7534-7540 (2001).

24. Y. Najima, N. Yahagi, Y. Takeuchi, T. Matsuzaka, M. Sekiya, Y. Nakagawa, M. Amemiya-Kudo, H. Okazaki, S. Okazaki, Y. Tamura, Y. Iizuka, K. Ohashi, K. Harada, T. Gotoda, R. Nagai, T. Kadowaki, S. Ishibashi, N. Yamada, J. I. Osuga, H. Shimano, High mobility group protein-B1 interacts with sterol regulatory element-binding proteins to enhance their DNA binding. J. Biol. Chem. 280, 27523-27532 (2005).

25. A. Agresti, P. Scaffidi, A. Riva, V. R. Caiolfa, M. E. Bianchi, GR and HMGB1 interact only within chromatin and influence each other's residence time. Mol. Cell. 18, 109-121 (2005).

26. S. Sofiadis, K. K., N. Nikolic, M. M., Z. Zirkel, A. A., K. Kargapolova, Y. Y., J. Josipovic, N. N., P. Papadakis, A. A., G. Gusmao, E. E., M. Mizi, A. A., G. Georgomanolis, T. T., K. Koker, M. M., U. Ullrich, R. R., A. Altmueller, J. J., N. Nuernberg, P. P., B. Beyer, A. A., P. Papantonis, A. A., HMGB1 as a rheostat of chromatin topology and RNA homeostasis on the path to senescence. bioRxiv Genomics, 1-33 (2019).

27. N. Senda, H. Yanai, S. Hibino, L. Li, Y. Mizushima, T. Miyagaki, M. Saeki, Y. Kishi, S. Hangai, J. Nishio, M. Sugaya, T. Taniguchi, S. Sato, HMGB1-mediated chromatin 
remodeling attenuates I124 gene expression for the protection from allergic contact dermatitis. Proc. Natl. Acad. Sci. U. S. A. 118 (2020), doi:10.1073/pnas.2022343118.

28. S. Calogero, F. Grassi, A. Aguzzi, T. Voigtländer, P. Ferrier, S. Ferrari, M. E. Bianchi, The lack of chromosomal protein Hmg1 does not disrupt cell growth but causes lethal hypoglycaemia in newborn mice. Nat. Genet. 22, 276-80 (1999).

29. P. Huebener, G.-Y. Gwak, J.-P. Pradere, C. M. Quinzii, R. Friedman, C.-S. Lin, C. M. Trent, I. Mederacke, E. Zhao, D. H. Dapito, Y. Lin, I. J. Goldberg, M. J. Czaja, R. F. Schwabe, High-mobility group box 1 is dispensable for autophagy, mitochondrial quality control, and organ function in vivo. Cell Metab. 19, 539-47 (2014).

30. T. Jelenik, K. Kaul, G. Séquaris, U. Flögel, E. Phielix, J. Kotzka, B. Knebel, P. Fahlbusch, T. Hörbelt, S. Lehr, A. L. Reinbeck, D. Müller-Wieland, I. Esposito, G. I. Shulman, J. Szendroedi, M. Roden, Mechanisms of insulin resistance in primary and secondary nonalcoholic fatty liver. Diabetes. 66, 2241-2253 (2017).

31. M. H. Oosterveer, T. H. Van Dijk, A. Grefhorst, V. W. Bloks, R. Havinga, F. Kuipers, D. J. Reijngoud, Lxro deficiency hampers the hepatic adaptive response to fasting in mice. J. Biol. Chem. 283, 25437-25445 (2008).

32. Y. Zhang, S. R. Breevoort, J. Angdisen, M. Fu, D. R. Schmidt, S. R. Holmstrom, S. A. Kliewer, D. J. Mangelsdorf, I. G. Schulman, Liver LXR $\alpha$ expression is crucial for whole body cholesterol homeostasis and reverse cholesterol transport in mice. J. Clin. Invest. 122, 1688-1699 (2012).

33. K. Matsusue, M. Haluzik, G. Lambert, S.-H. Yim, O. Gavrilova, J. M. Ward, B. Brewer, M. L. Reitman, F. J. Gonzalez, Liver-specific disruption of PPAR $\gamma$ in leptin-deficient mice improves fatty liver but aggravates diabetic phenotypes. J. Clin. Invest. 111, $737-$ 747 (2003).

34. E. Morán-Salvador, M. López-Parra, V. García-Alonso, E. Titos, M. MartínezClemente, A. González-Périz, C. López-Vicario, Y. Barak, V. Arroyo, J. Clària, Role for PPAR $\gamma$ in obesity-induced hepatic steatosis as determined by hepatocyte- and macrophage-specific conditional knockouts. FASEB J. 25, 2538-2550 (2011).

35. A. B. Engin, in Advances in Experimental Medicine and Biology (Springer New York LLC, 2017), vol. 960, pp. 197-220.

36. J. Zhang, L. Zhang, S. Zhang, Q. Yu, F. Xiong, K. Huang, C. Y. Wang, P. Yang, HMGB1, an innate alarmin, plays a critical role in chronic inflammation of adipose tissue in obesity. Mol. Cell. Endocrinol. 454 (2017), pp. 103-111.

37. D. Bertheloot, E. Latz, HMGB1, IL-1 $\alpha$, IL-33 and S100 proteins: Dual-function alarmins. Cell. Mol. Immunol. 14 (2017), pp. 43-64.

38. T. Shimizu, M. Yamakuchi, K. K. Biswas, B. Aryal, S. Yamada, T. Hashiguchi, I. Maruyama, HMGB1 is secreted by 3T3-L1 adipocytes through JNK signaling and the secretion is partially inhibited by adiponectin. Obesity. 24, 1913-1921 (2016).

39. M. K. Gunasekaran, W. Viranaicken, A.-C. Girard, F. Festy, M. Cesari, R. Roche, L. Hoareau, Inflammation triggers high mobility group box 1 (HMGB1) secretion in adipose tissue, a potential link to obesity. Cytokine. 64, 103-11 (2013).

40. L. Jia, H. Song, W. Fan, Y. Song, G. Wang, X. Li, Y. He, A. Yao, The association between high mobility group box 1 chromatin protein and mitotic chromosomes in glioma cells. Oncol. Lett. 19, 745-752 (2020).

41. T. T. Schug, X. Li, Sirtuin 1 in lipid metabolism and obesity. Ann. Med. 43, 198-211 (2011).

42. S. Xu, Z. Zeng, M. Zhao, Q. Huang, Y. Gao, X. Dai, J. Lu, W. Huang, K. Zhao, Evidence for SIRT1 Mediated HMGB1 Release From Kidney Cells in the Early Stages of Hemorrhagic Shock. Front. Physiol. 10, 854 (2019).

43. S. Ghaffari, E. Jang, F. N. Nabi, R. Sanwal, N. Khosraviani, C. Wang, B. E. Steinberg, 
N. M. Goldenberg, J. Ikeda, W. L. Lee, Endothelial HMGB1 Is a Critical Regulator of LDL Transcytosis via an SREBP2-SR-BI Axis. Arterioscler. Thromb. Vasc. Biol. (2020), doi:10.1161/atvbaha.120.314557.

44. M. Fortier, S. Celton-Morizur, C. Desdouets, Incomplete cytokinesis/binucleation in mammals: The powerful system of hepatocytes. Methods Cell Biol. 137, 119-142 (2017).

45. C. Laurens, V. Bourlier, A. Mairal, K. Louche, P. M. Badin, E. Mouisel, A. Montagner, A. Marette, A. Tremblay, J. S. Weisnagel, H. Guillou, D. Langin, D. R. Joanisse, C. Moro, Perilipin 5 fine-tunes lipid oxidation to metabolic demand and protects against lipotoxicity in skeletal muscle. Sci. Rep. 6 (2016), doi:10.1038/srep38310.

46. R: The R Project for Statistical Computing, (available at https://www.r-project.org/).

47. W. Huber, V. J. Carey, R. Gentleman, S. Anders, M. Carlson, B. S. Carvalho, H. C. Bravo, S. Davis, L. Gatto, T. Girke, R. Gottardo, F. Hahne, K. D. Hansen, R. A. Irizarry, M. Lawrence, M. I. Love, J. MaCdonald, V. Obenchain, A. K. Oles̈, H. Pagès, A. Reyes, P. Shannon, G. K. Smyth, D. Tenenbaum, L. Waldron, M. Morgan, Orchestrating highthroughput genomic analysis with Bioconductor. Nat. Methods. 12, 115-121 (2015).

48. B. M. Bolstad, R. A. Irizarry, M. Åstrand, T. P. Speed, A comparison of normalization methods for high density oligonucleotide array data based on variance and bias. Bioinformatics. 19, 185-193 (2003).

49. M. E. Ritchie, B. Phipson, D. Wu, Y. Hu, C. W. Law, W. Shi, G. K. Smyth, Limma powers differential expression analyses for RNA-sequencing and microarray studies. Nucleic Acids Res. 43, e47 (2015).

50. Z. Gu, R. Eils, M. Schlesner, Complex heatmaps reveal patterns and correlations in multidimensional genomic data. Bioinformatics. 32, 2847-2849 (2016).

51. S. Durinck, P. T. Spellman, E. Birney, W. Huber, Mapping identifiers for the integration of genomic datasets with the R/ Bioconductor package biomaRt. Nat. Protoc. 4, 11841191 (2009).

52. G. Sales, E. Calura, D. Cavalieri, C. Romualdi, Graphite - a Bioconductor package to convert pathway topology to gene network. BMC Bioinformatics. 13, 1-12 (2012).

53. M. V. Kuleshov, M. R. Jones, A. D. Rouillard, N. F. Fernandez, Q. Duan, Z. Wang, S. Koplev, S. L. Jenkins, K. M. Jagodnik, A. Lachmann, M. G. McDermott, C. D. Monteiro, G. W. Gundersen, A. Ma'ayan, Enrichr: a comprehensive gene set enrichment analysis web server 2016 update. Nucleic Acids Res. 44, W90-W97 (2016).

54. H. Wickham, ggplot2 (Springer New York, 2009).

55. P. Shannon, A. Markiel, O. Ozier, N. S. Baliga, J. T. Wang, D. Ramage, N. Amin, B. Schwikowski, T. Ideker, Cytoscape: A software Environment for integrated models of biomolecular interaction networks. Genome Res. 13, 2498-2504 (2003).

56. J. D. Buenrostro, P. G. Giresi, L. C. Zaba, H. Y. Chang, W. J. Greenleaf, Transposition of native chromatin for fast and sensitive epigenomic profiling of open chromatin, DNAbinding proteins and nucleosome position. Nat. Methods. 10, 1213-1218 (2013).

57. M. R. Corces, A. E. Trevino, E. G. Hamilton, P. G. Greenside, N. A. Sinnott-Armstrong, S. Vesuna, A. T. Satpathy, A. J. Rubin, K. S. Montine, B. Wu, A. Kathiria, S. W. Cho, M. R. Mumbach, A. C. Carter, M. Kasowski, L. A. Orloff, V. I. Risca, A. Kundaje, P. A. Khavari, T. J. Montine, W. J. Greenleaf, H. Y. Chang, An improved ATAC-seq protocol reduces background and enables interrogation of frozen tissues. Nat. Methods. 14, 959962 (2017).

58. B. Langmead, S. L. Salzberg, Fast gapped-read alignment with Bowtie 2. Nat. Methods. 9, 357-359 (2012).

59. Y. Zhang, T. Liu, C. A. Meyer, J. Eeckhoute, D. S. Johnson, B. E. Bernstein, C. Nussbaum, R. M. Myers, M. Brown, W. Li, X. S. Shirley, Model-based analysis of ChIPSeq (MACS). Genome Biol. 9, R137 (2008). 
60. G. Yu, L. G. Wang, Q. Y. He, ChIP seeker: An R/Bioconductor package for ChIP peak annotation, comparison and visualization. Bioinformatics. 31, 2382-2383 (2015).

61. M. I. Love, W. Huber, S. Anders, Moderated estimation of fold change and dispersion for RNA-seq data with DESeq2. Genome Biol. 15, 550 (2014).

62. O. Fornes, J. A. Castro-Mondragon, A. Khan, R. Van Der Lee, X. Zhang, P. A. Richmond, B. P. Modi, S. Correard, M. Gheorghe, D. Baranašić, W. Santana-Garcia, G. Tan, J. Chèneby, B. Ballester, F. Parcy, A. Sandelin, B. Lenhard, W. W. Wasserman, A. Mathelier, JASPAR 2020: Update of the open-Access database of transcription factor binding profiles. Nucleic Acids Res. 48, D87-D92 (2020).

63. G. Yu, Q. Y. He, ReactomePA: An R/Bioconductor package for reactome pathway analysis and visualization. Mol. Biosyst. 12, 477-479 (2016).

\section{Acknowledgments}

The authors would like to thank the phenotyping facility (Anexplo-US006/CREFRE, Toulouse, France) for all plasma analysis and technical assistance. We also thank the Histology (Lucie Fontaine) and the Functional Biochemistry (Alexandre Lucas) Facilities at the i2MC for technical assistance (UMR1048-Toulouse, France). We thank Claire Naylies and Yannick Lippi for their contribution to microarray fingerprints acquisition and microarray data analysis carried out at GeT Genopole Toulouse Midi-Pyrénées facility (https://doi.org/10.15454/1.5572370921303193E12). Jean-José Maoret (GeT-Santé, UMR1048Toulouse, France) for his technical help during the microfluidic PCR experiments, Justine Bertrand-Michel and the lipidomic facility (Genotoul-MetaToul Lipidomique, UMR1048Toulouse, France) for all lipid analysis and technical assistance. We kindly thank Dr. PierreDamien Denechaud (i2MC-INSERM-Toulouse) for fruitful discussions and kind advices.

\section{Funding:}

This study is supported by grants from INSERM, Paul Sabatier University, the Agence Nationale de la Recherche (ANR-17-CE14-0016, J-PP) and Association Française d'Etude et de Recherche sur l'Obésité (J-PP). JP is supported by a scholarship from Paul Sabatier University, EP is supported by a scholarship from Agence Nationale de la Recherche (ANR-17-CE140016) and RP is supported by a scholarship from Région Midi-Pyrénées-INSERM $\left(n^{\circ} 15050341\right)$.

\section{Author contributions}

JP designed research, performed all experiments, analyzed data and wrote the manuscript; AD, AP, AB, SD, EP, RP and EM performed experiments and analyzed data, J.SI and JM analyzed high-throughput data and draft related figures. TC and GL carried out the ChIP-seq 
experiments, analyzed data and reviewed the manuscript. AM and W.AW generously provided $\operatorname{PPAR} \gamma^{\mathrm{fl} / \mathrm{fl}}$ and PPAR $\gamma^{\Delta \mathrm{Hep}}$ mice. W.AW reviewed and commented on the manuscript. R.FS kindly provided HMGB1 floxed mice and reviewed the manuscript. AY and IC-L have provided valuable inputs, daily support, have reviewed and edited the manuscript. $\mathrm{CP}$ and FB kindly provided the adenovirus expressing shRNA-targeting LXR, reviewed and commented on the manuscript; CM performed lipogenesis and Beta-Ox assays in vitro and in vivo, analyzed data and reviewed and commented on the manuscript; HG designed and performed experiments, analyzed data provided reagents, gave important input related to the study design, reviewed and commented on the manuscript, PV and CD have provided daily support, fruitful discussions, 1080 fundings and revised and commented on the manuscript; J-PP conceived the original 1081 hypothesis, designed all experiments, performed experiments, analyzed data, wrote the manuscript, provided fundings and supervised the project.

1083

1084

\section{Competing interests:}

1085 The authors declare no conflicts of interest.

1086

1087

Data and materials availability: All data needed to evaluate the conclusions in the paper are 1088 present in the paper and/or the Supplementary Materials.

1089

1090 
A.
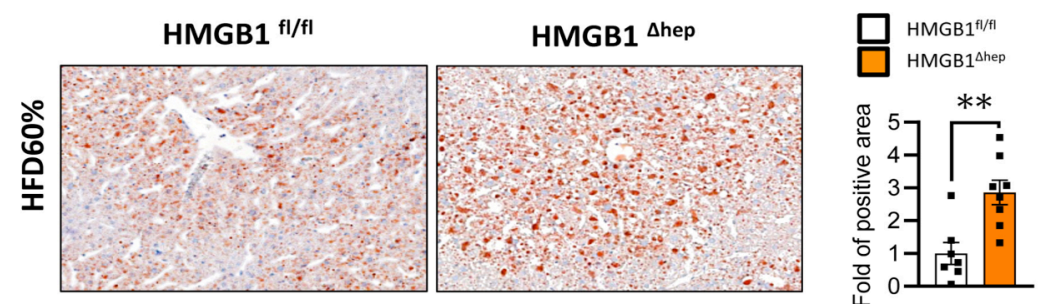

B.
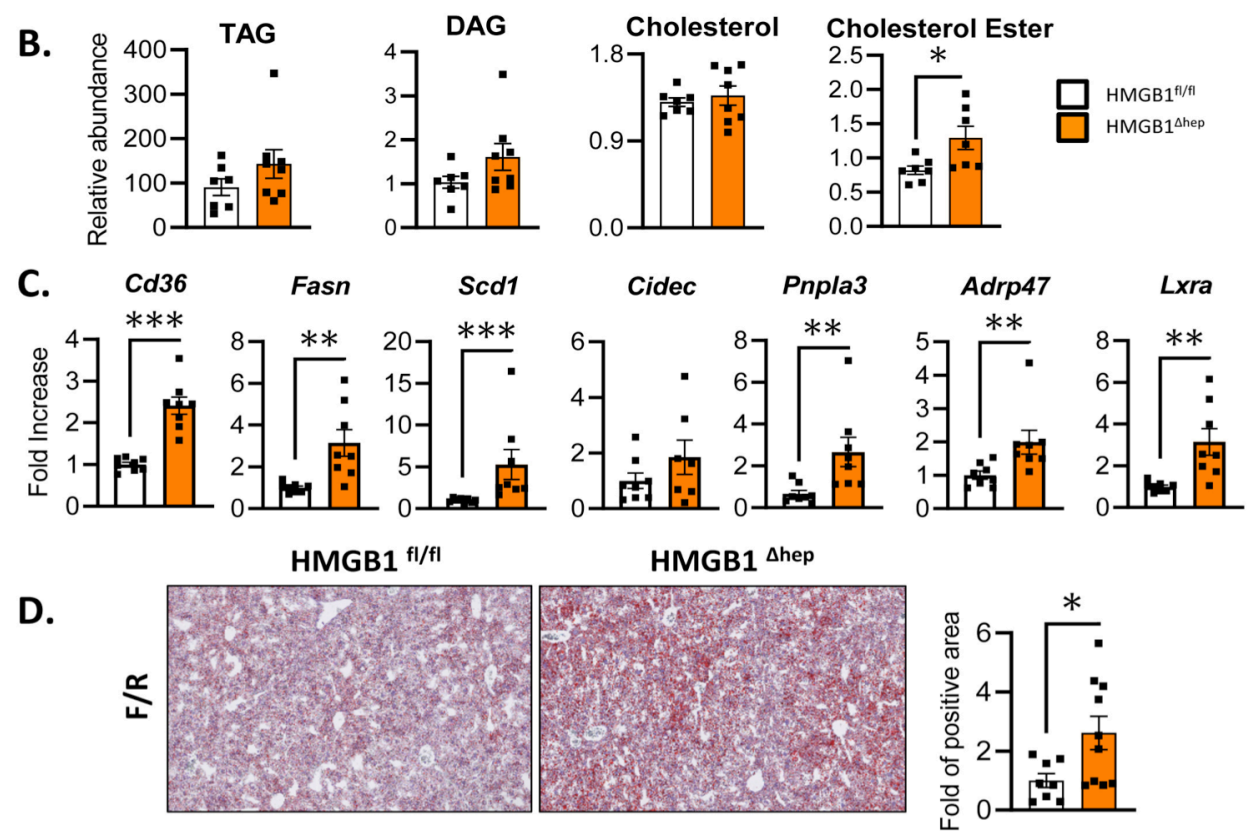

E.
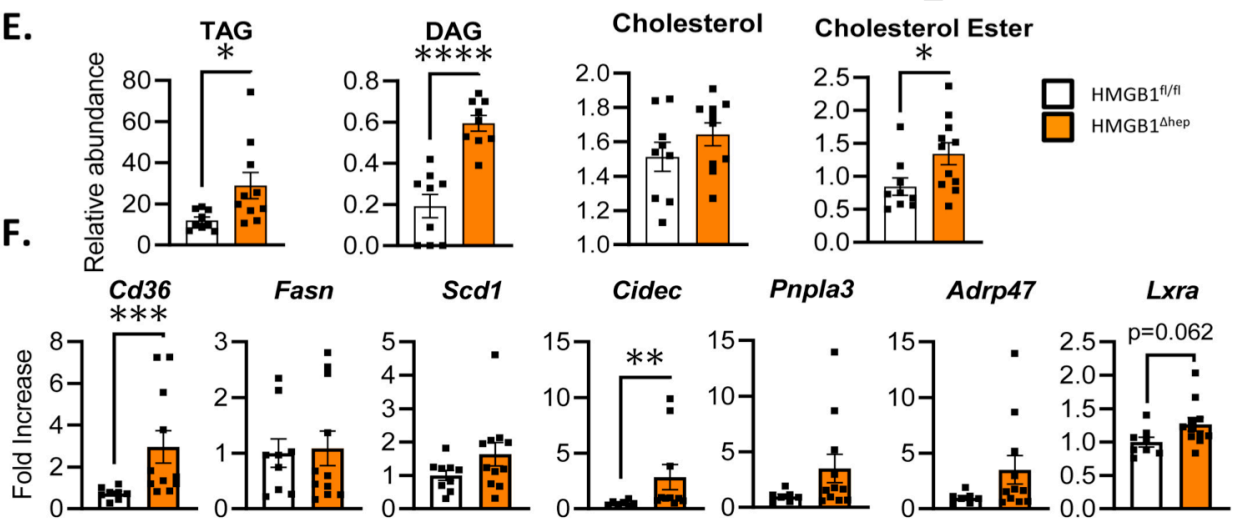

Fig. 1. Hepatocyte specific Hmgb1 deleted mice on HFD or after fasting/refeeding challenge exhibit a severe liver steatosis.

(A) Oil Red-O staining on liver section with quantification, (B) neutral lipid analysis and (C) mRNA expression of hepatic steatosis markers from liver biopsies of $\mathrm{HMGB1} 1^{\mathrm{fl} / \mathrm{fl}}$ and HMGB1 ${ }^{\Delta \text { Hep }}$ mice subjected to 12 -week HFD. (D) Oil Red-O staining on liver section with quantification, $(\mathbf{E})$ neutral lipid analysis and (F) mRNA expression of hepatic steatosis markers from liver biopsies of $\mathrm{HMGB}^{\mathrm{fl} / \mathrm{fl}}$ and $\mathrm{HMGB} 1^{\Delta \mathrm{Hep}}$ mice after a fasting/refeeding challenge. Data are means \pm SEM from $n=7\left(\mathrm{HMGB}^{\mathrm{fl} / \mathrm{fl}}\right)$ or $\mathrm{n}=8\left(\mathrm{HMGB}{ }^{\Delta \mathrm{Hep}}\right)$ per group for the HFD protocol $(\mathbf{A}-\mathbf{C})$ and from $n=8\left(\mathrm{HMGB}^{\mathrm{fl} / \mathrm{fl}}\right)$ or $\mathrm{n}=8\left(\mathrm{HMGB}^{\Delta \mathrm{Hep}}\right)$ per group for the $\mathrm{F} / \mathrm{R}$ protocol (D-F). ${ }^{*} p<0.05, * * p<0.01, * * * p<0.001, * * * * p<0.0001$ by unpaired Mann and Whitney comparison. 

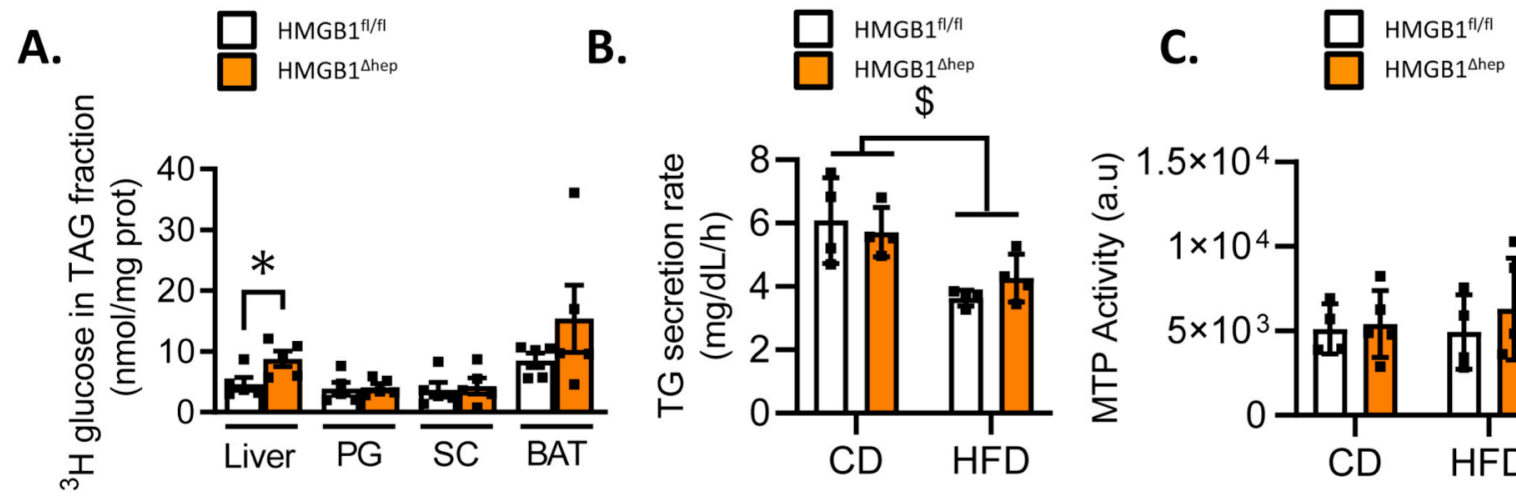

D.

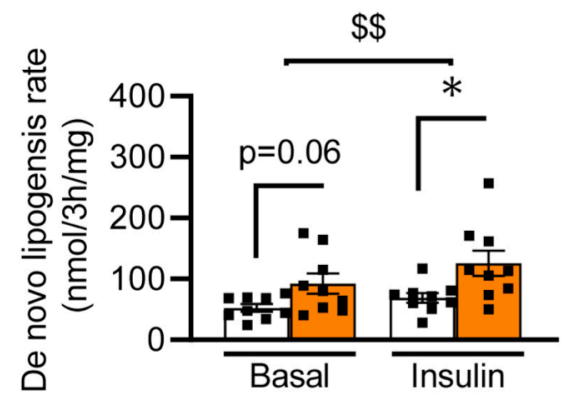

E.
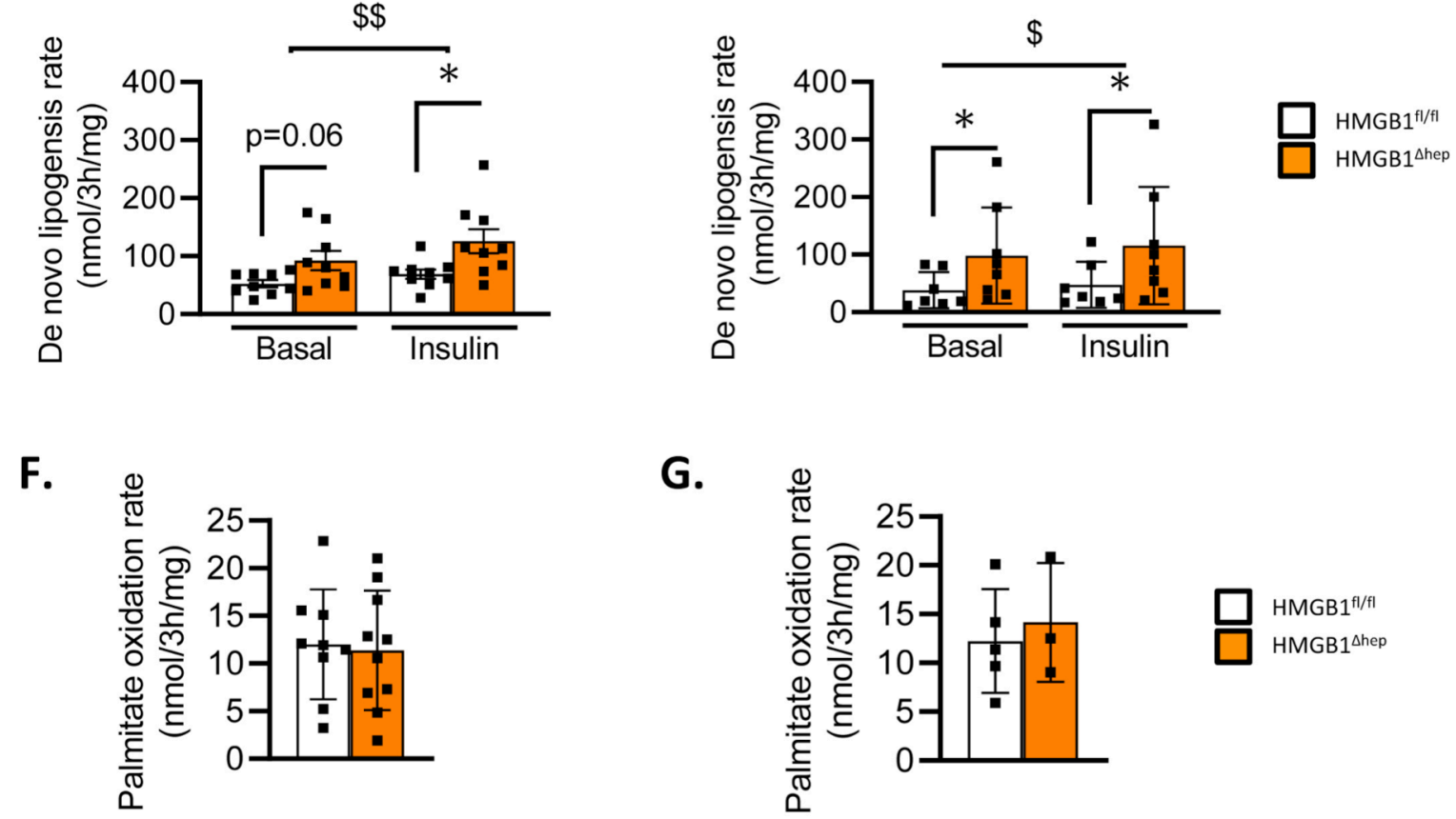

G.

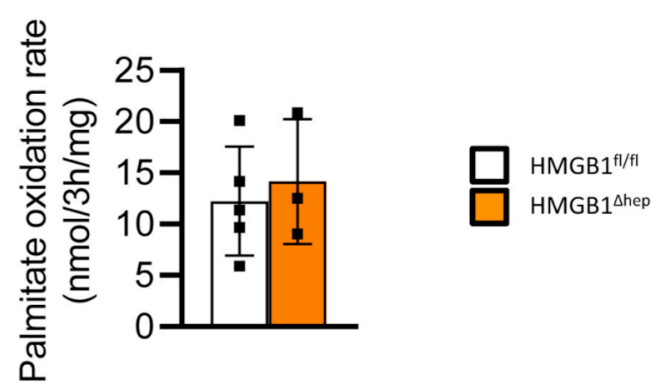

Fig. 2. Hmgb1 deletion increases hepatocyte lipid synthesis in vitro and in vivo.

(A) In vivo, lipogenesis was measured on $\mathrm{HMGB}^{\mathrm{fl} / \mathrm{fl}}(\mathrm{n}=5)$ and $\mathrm{HMGB}^{\Delta \mathrm{Hep}}(\mathrm{n}=5)$ mice. Mice were food deprived for six hours then injected with ${ }^{3} \mathrm{H}$ glucose $(0.4 \mu \mathrm{Ci} / \mathrm{g}$, i.p) and euthanized one hour later and ${ }^{3} \mathrm{H}$ was measured in TAG fraction of liver, adipose tissues (PG, SC and BAT). (B-C) In vivo, assessment of liver lipoprotein secretions determined by (B) measuring circulating tri-acyl glycerol concentration $(\mathrm{n}=4$ per genotype and diet) and (C) liver MTP activity, $\mathrm{HMGB}^{\mathrm{fl} / \mathrm{fl}}(\mathrm{n}=4)$ and $\mathrm{HMGB} 1^{\Delta \mathrm{Hep}}(\mathrm{n}=5)$. (D-E) Lipid synthesis was measured in vitro, on primary hepatocytes isolated from adult HMGB1 ${ }^{\mathrm{fl} / \mathrm{fl}}(\mathrm{n}=7-9)$ and HMGB1 ${ }^{\Delta \mathrm{Hep}}(\mathrm{n}=8-9)$ mice on (D) chow diet and (E) HFD. (F-G) Beta-oxidation was measured in vitro, on primary hepatocytes isolated from adult HMGB1 $1^{\mathrm{f} / \mathrm{fl}}$ and $\mathrm{HMGB} 1^{\Delta \mathrm{Hep}}$ mice on $(\mathbf{F})$ chow diet $\left(\mathrm{HMGB} 1^{\mathrm{fl} / \mathrm{fl}}\right.$ $\mathrm{n}=9$ and $\mathrm{HMGB}^{\Delta \mathrm{Hep}} \mathrm{n}=10$ ) and (G) HFD $\left(\mathrm{HMGB}^{\mathrm{fl} / \mathrm{fl}} \mathrm{n}=5\right.$ and HMGB1 ${ }^{\Delta \mathrm{Hep}} \mathrm{n}=3$ ). Data are means \pm SEM of three independent experiments. $p<0.05, * * p<0.01, * * * p<0.001, * * * * p<0.0001$ by unpaired Mann and Whitney comparison or two-way ANOVA. $\$ \mathrm{p}<0.05, \$ \$ \mathrm{p}<0.01, \$ \$ \$$ $\mathrm{p}<0.001$, for treatment effect by one-way ANOVA. 
A.

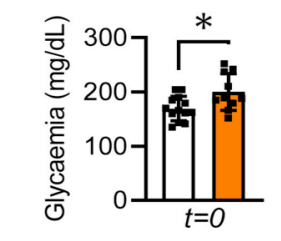

F.

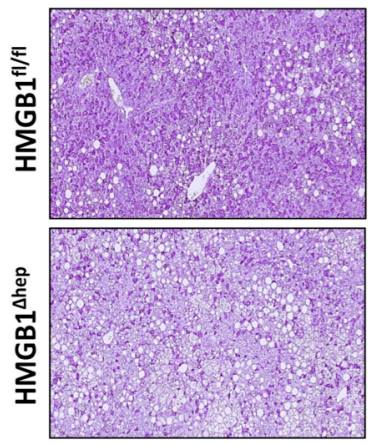

G.

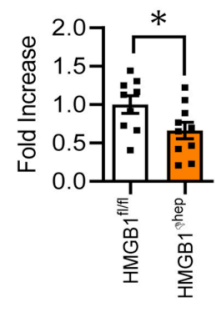

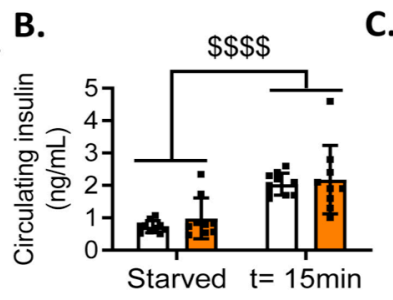

E.

E. Pyruvate Tolerance Test

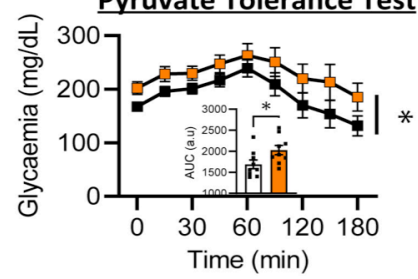

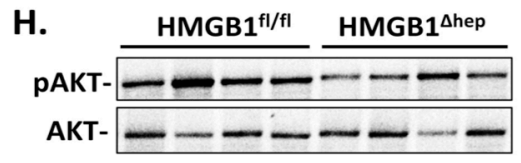

I.

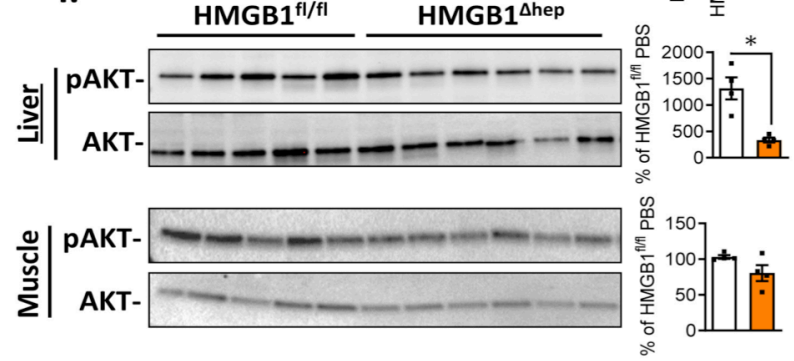

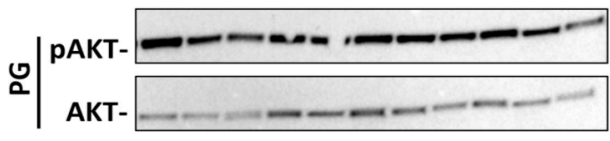

C. Insulin Tolerance Test

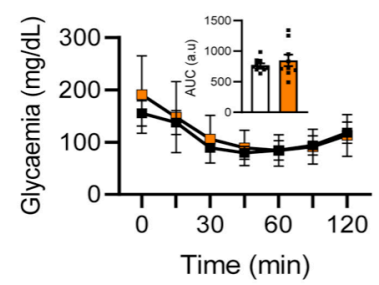

$\square \mathrm{HMGB}^{\mathrm{fl} / \mathrm{fl}}$

Fig. 3. Hepatocyte specific $\mathbf{H m g b 1}$ deleted mice on HFD display reduced insulin sensitivity in the liver.

(A) Analysis of oral glucose tolerance test, (B) Insulin levels after fasting or 15 minutes post glucose bolus, (C) insulin tolerance test (D) Fasting glycaemia levels after 16 hours of fasting. and (E) pyruvate tolerance test on HMGB1 $1^{\mathrm{fl} / \mathrm{fl}}$ and $\mathrm{HMGB}^{\Delta \mathrm{Hep}}$ mice fed on HFD for 12 weeks. (F) Hepatic PAS staining representative images with $(\mathbf{G})$ quantification on HMGB1 ${ }^{\mathrm{fl} / \mathrm{fl}}$ and HMGB1 $1^{\Delta \mathrm{Hep}}$ mice fed on HFD for 12 weeks. (H) Representative immunoblot targeting p-AKT and tot-AKT with quantification performed on the whole animal cohort, on liver biopsies from on HMGB1 $1^{\text {fl/fl }}$ and HMGB1 $1^{\Delta \text { Hep }}$ mice fed on HFD for 12 weeks. (I) Representative immunoblot targeting p-AKT and tot-AKT with quantification performed on the whole animal cohort, on liver biopsies from $\mathrm{HMGB}_{1}^{\mathrm{fl} / \mathrm{fl}}$ and $\mathrm{HMGB} 1^{\Delta \mathrm{Hep}}$ mice fed on HFD for 24 weeks, starved 4 hours and injected with insulin (i.p. $0.75 \mathrm{U} / \mathrm{kg}-15$ minutes). Data are means \pm SEM from $\mathrm{n}=10$ $\left(\mathrm{HMGB1}^{\mathrm{fl} / \mathrm{fl}}\right.$ ) or $\mathrm{n}=11\left(\mathrm{HMGB}^{\Delta \mathrm{Hep}}\right)$ per group for the HFD protocol $(\mathrm{A}-\mathrm{H})$ and from $\mathrm{n}=4$ $\left(\mathrm{HMGB}^{\mathrm{fl} / \mathrm{fl}}\right)$ or $\mathrm{n}=4\left(\mathrm{HMGB}^{\Delta \mathrm{Hep}}\right)$ per group for the HFD 24-week with acute injection of insulin protocol (I). ${ }^{*} \mathrm{p}<0.05,{ }^{*} \mathrm{p}<0.01,{ }^{* * *} \mathrm{p}<0.001,{ }^{* * * *} \mathrm{p}<0,0001$ by unpaired Mann and Whitney comparison or two-way ANOVA. 


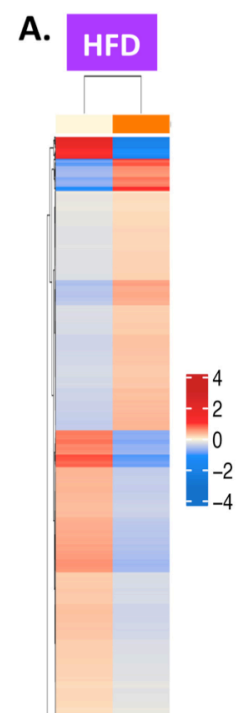

D. Gene Ontology

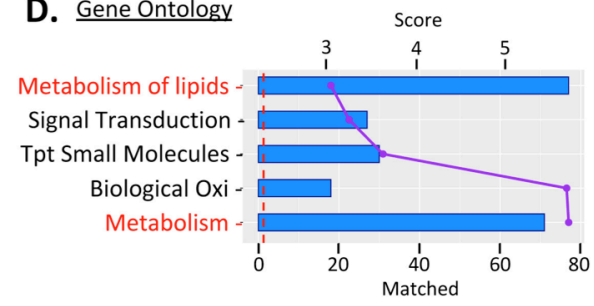

F.

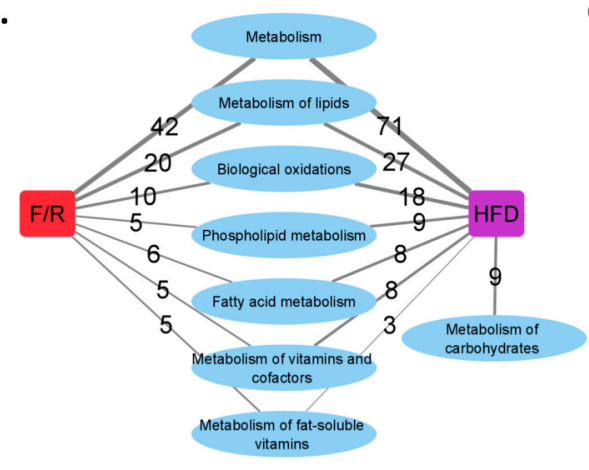

B.
C.
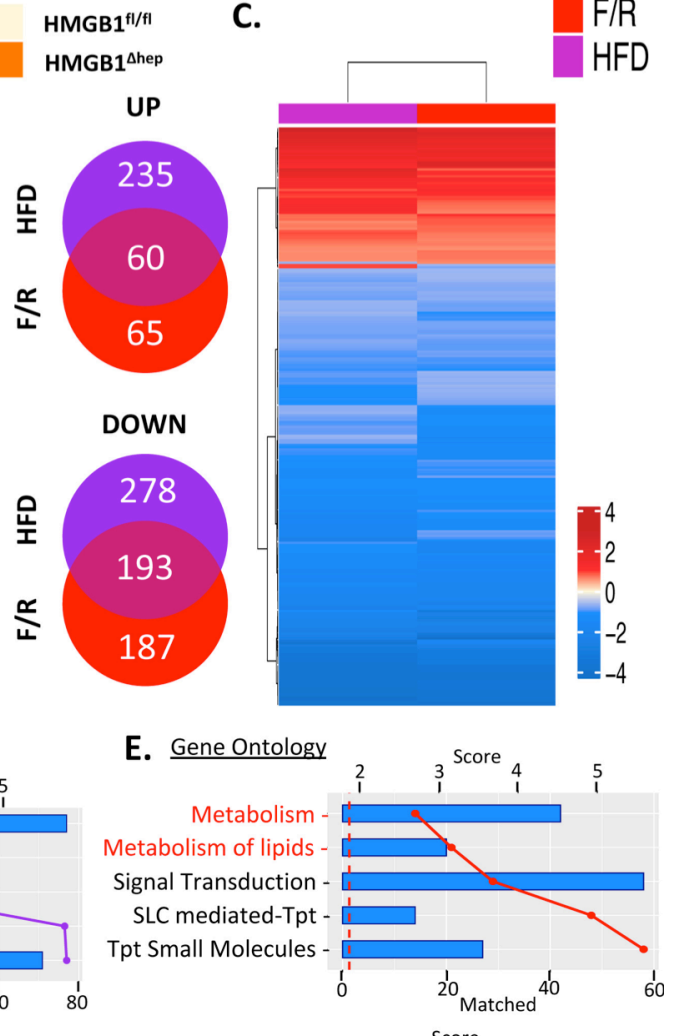

G.

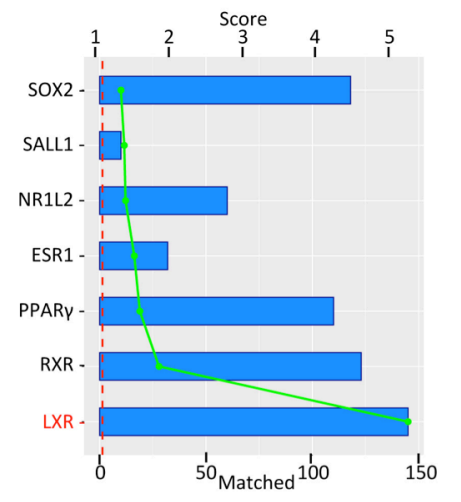

Fig. 4. Microarray analysis of hepatic gene expression profiles in $\mathrm{HMGB}^{\mathrm{\Delta Hep}}$ mice.

(A) Heatmap showing genes that are differentially expressed in the livers of HMGB $1^{\triangle \mathrm{Hep}}$ mice compared to HMGB1 ${ }^{\mathrm{fl} / \mathrm{fl}}$ mice (fold change $>1.5$; P-Value $<=0.01$ ) after HFD (left panel) or F/R (right panel). Heatmaps display the mean normalized expression per genotype per nutritional challenge. (B) Venn Diagram displaying overlap between up and down regulated genes in the two regimens. (C) Heatmap displaying only DEG commonly found in both regimens (fold change $>1.5$; P-Value $<=0.01$ ). (D-E) Top $5 \mathrm{GO}$ biological processes enriched using gene sets for each regimen, with the $-\log 10$ (P-Value) of enrichment shown as bars and the number of matched genes as colored lines. (F) Network displaying Reactome pathways related to metabolism that are enriched by our HMGB1 gene sets from both nutritional challenges, edge thickness represents the number of genes regulated by HMGB1 among each sub-category. (G) Top upstream regulators identified using the ChEA database, with the -log10(P-Value) of enrichment as bars and the number of gene matched per as a green line. Data are means \pm SEM from $n=4\left(\mathrm{HMGB}^{\mathrm{fl} / \mathrm{fl}}\right)$ or $\mathrm{n}=4\left(\mathrm{HMGB} 1^{\Delta \mathrm{Hep}}\right)$ per group for the 12 week-HFD protocol and from $\mathrm{n}=4\left(\mathrm{HMGB}^{\mathrm{fl} / \mathrm{fl}}\right)$ or $\mathrm{n}=4\left(\mathrm{HMGB} 1^{\Delta \mathrm{Hep}}\right)$ per group for the $\mathrm{F} / \mathrm{R}$ protocol. 

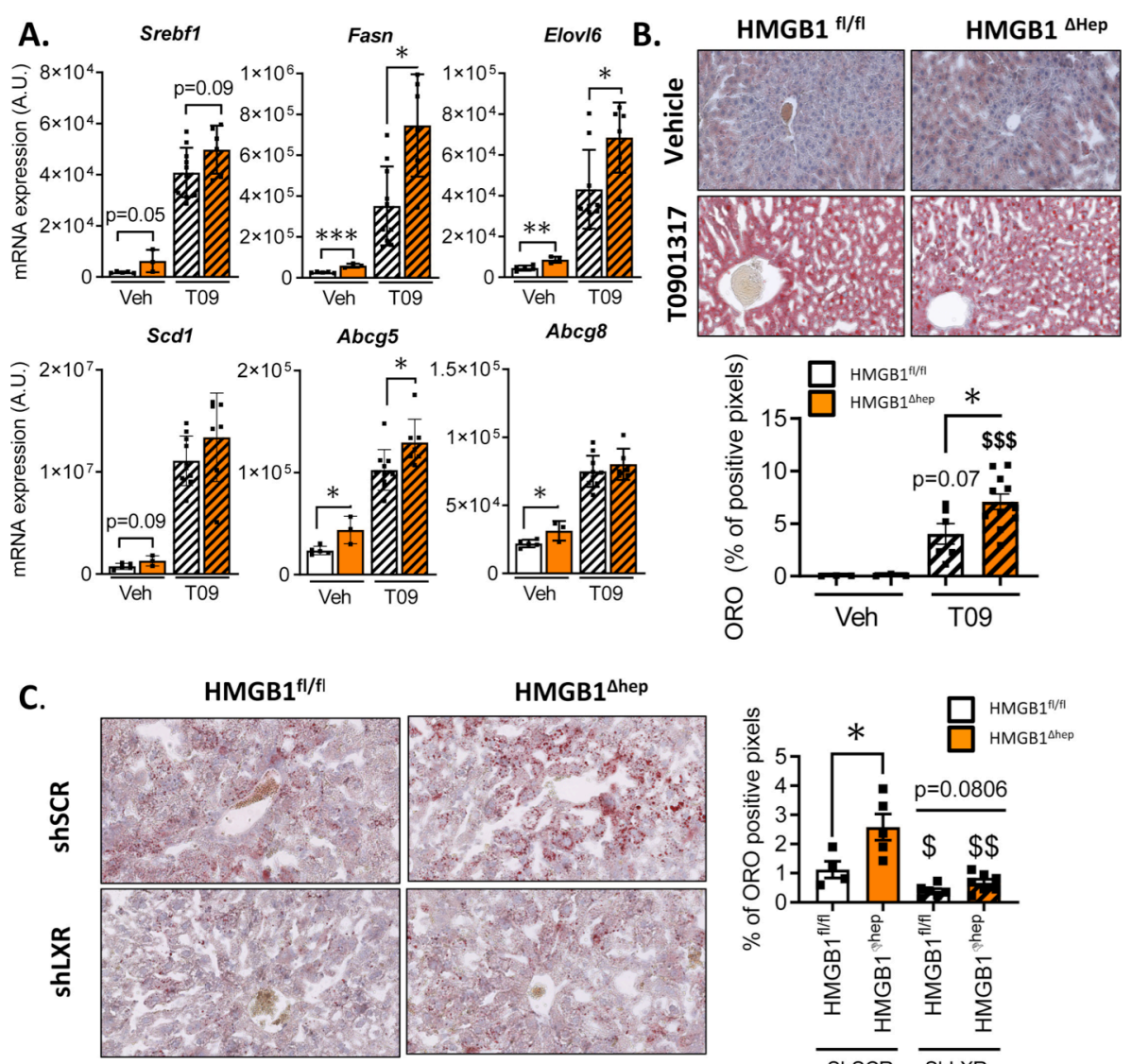

D.
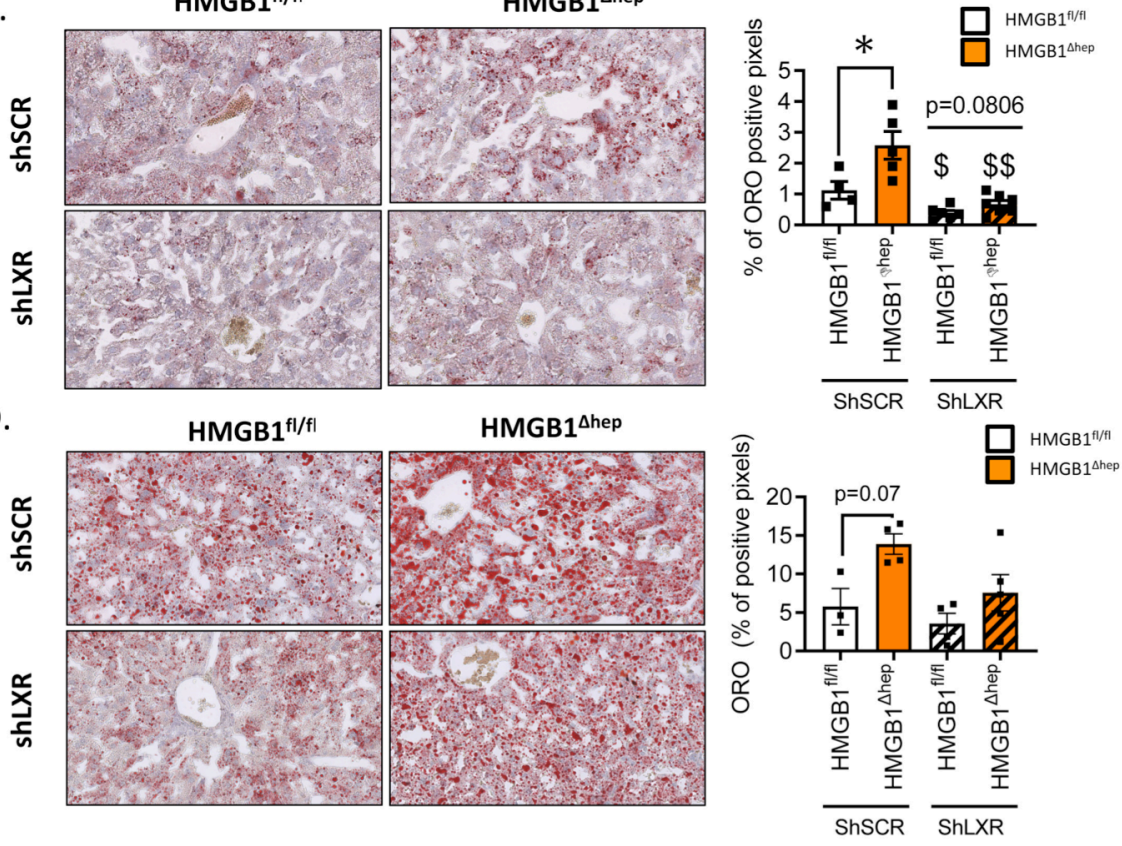

\section{Fig. 5. In vivo knockdown of LXR normalizes liver steatosis in HMGB1 ${ }^{\Delta \mathrm{Hep}}$ mice. $^{-}$}

(A-B) $\operatorname{HMGB}^{\mathrm{fl} / \mathrm{fl}}(\mathrm{n}=15)$ and $\mathrm{HMGB}^{\Delta \mathrm{Hep}}(\mathrm{n}=9)$ mice were treated either with vehicle $(5 \%$ carboxy-methyl-cellulose) or LXR synthetic agonist T0901317 (oral gavage, $30 \mathrm{mg} / \mathrm{kg} / \mathrm{day}$ ) for four consecutive days, after 6 hours starvation on the last day mice were sacrificed. (A) Liver tissue was then subjected to RT-qPCR analysis of the indicated LXR dependent genes and (B) liver steatosis was quantified using Oil Red-O staining. (C) HMGB1 ${ }^{\mathrm{fl} / \mathrm{fl}} \quad(\mathrm{n}=10)$ and $\mathrm{HMGB1}^{\Delta \mathrm{Hep}}(\mathrm{n}=12)$ mice were infected with either adenovirus expressing a LXR shRNA or a scramble (SCR) sequence, then subjected 7 days later to a $\mathrm{F} / \mathrm{R}$ challenge. Liver steatosis was determined by Oil Red-O staining on liver sections with the quantitative representation displayed on the right. (D) $H M G B 1^{\mathrm{fl} / \mathrm{fl}}$ and $\mathrm{HMGB} 1^{\mathrm{H} e \mathrm{p}}$ mice were subjected to HFD for four weeks and then infected with either adenovirus expressing a $\operatorname{LXR} \alpha \operatorname{shRNA}(n=7)$ or a scramble shRNA (SCR) $n=9$ ) sequence and euthanized 7 days later. Liver steatosis was assessed by Oil Red-O staining on liver section tissue with the quantitative representation displayed on the right. Data are means \pm SEM. ${ }^{*} p<0.05, * * p<0.01, * * * p<0.001, * * * * p<0.0001 \mathrm{HMGB} 1^{\mathrm{fl} / f \mathrm{l}}$ and $\mathrm{HMGB}^{\Delta \mathrm{Hep}}$ comparison, by unpaired Mann and Whitney comparison. $\$ \mathrm{p}<0.05, \$ \$ \mathrm{p}<0.01$, $\$ \$ \$ p<0.001$, for treatment effect by one-way ANOVA. 
A.

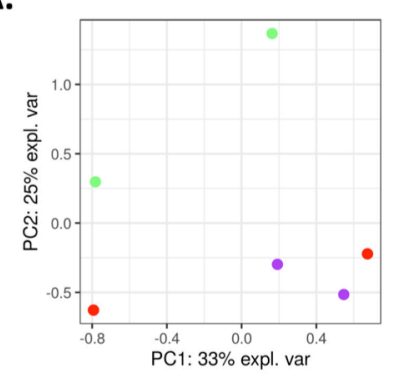

B.

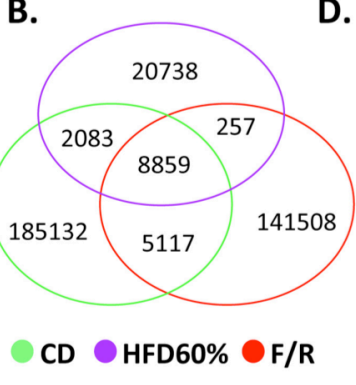

c. ans

$50 \mathrm{Mb}$

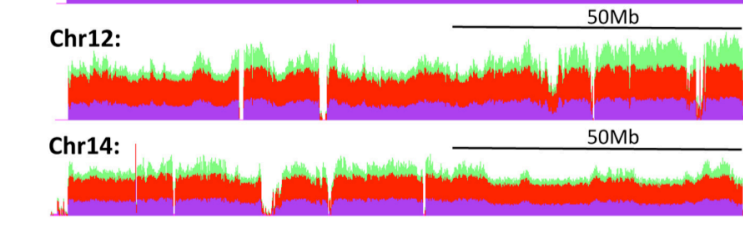

E.

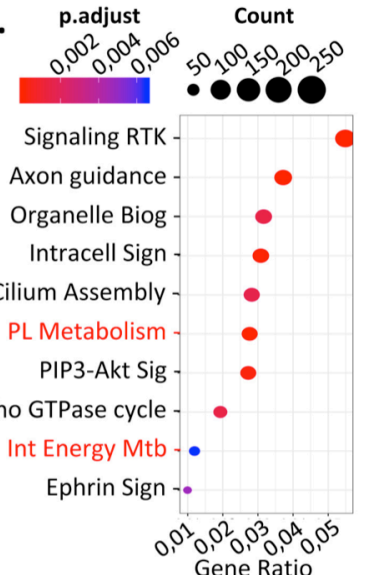

F. p.adjust

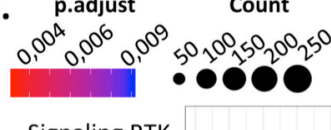

I.

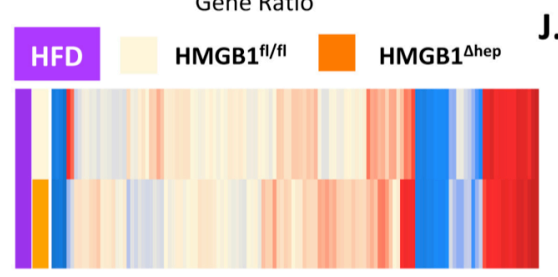

D. CD HFD60\% $\quad F / R$

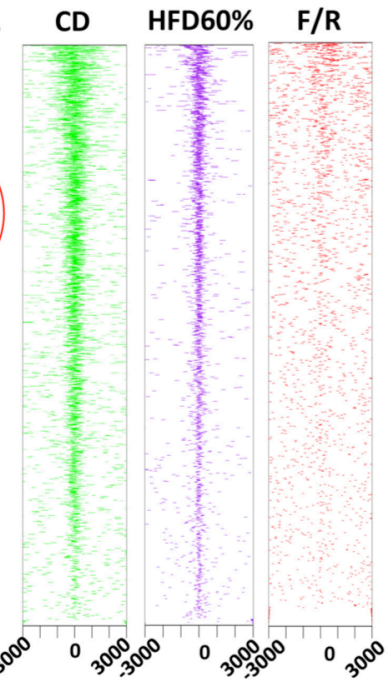

G.

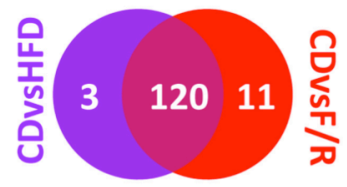

H.

Motif Analysis

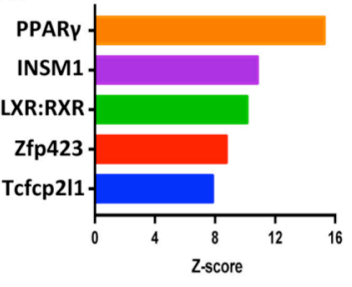

Fig. 6. ChIP-seq identified a subset of LXR responsive genes to be negatively regulated by HMGB1 during liver steatosis.

(A) Principal component analysis scores plot of ChIP-seq data of liver tissue from HMGB1 $1^{\mathrm{fl} / \mathrm{fl}}$ mice on chow diet (green) or subjected to F/R (red) or HFD (purple). (B) Venn Diagram showing the number of HMGB1 binding peaks, (C) UCSC genome browser of tracks (stacked) showing HMGB1 differential chromatin occupancy and (D) average signal density profiles around transcription starting site in different nutritional states: chow diet (green) or during HFD (purple) or after F/R (red). (E-F) Functional enrichment analyses showing GO terms associated with the differential HMGB1chromatin binding sites between (E) chow diet and HFD and (F) chow diet and F/R. (G) Venn Diagram displaying shared enriched genes $(n=134)$ displaying a very high occupancy rate during fed state belonging to "Integration of energy metabolism" and "Phospholipid metabolism" GO functions compared to HFD (purple) and F/R (red). (H) Graph bar displaying consensus motifs in promoters of the 134 genes differentially occupied by HMGB1 via OPOSUM analysis; the bars represent the z-score. (I-J) Heatmaps displaying the mean microarray expression levels for the 134 genes identified by ChIP-seq in liver from $\operatorname{HMGB1}^{\mathrm{fl} / \mathrm{fl}}(\mathrm{n}=4)$ and HMGB1 ${ }^{\Delta \mathrm{Hep}}(\mathrm{n}=4)$ mice subjected to either HFD (I) or F/R (J). 
A.

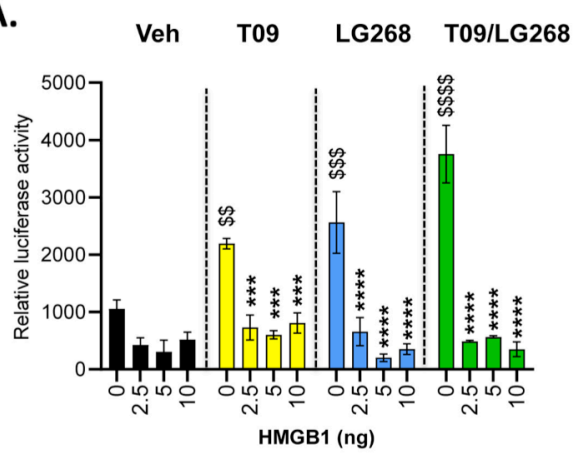

C.

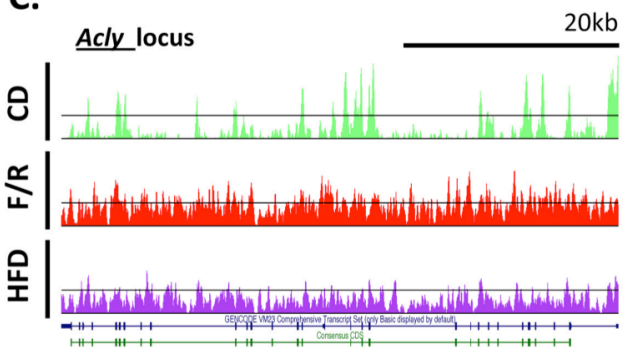

B.

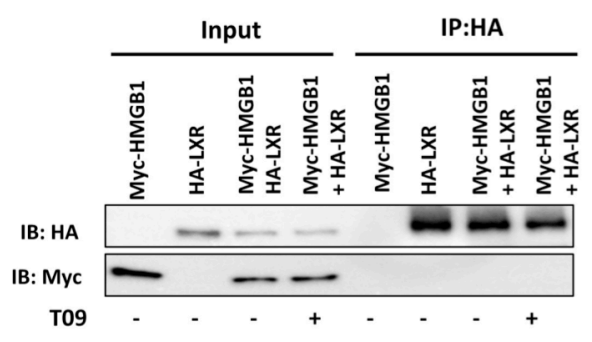

D.

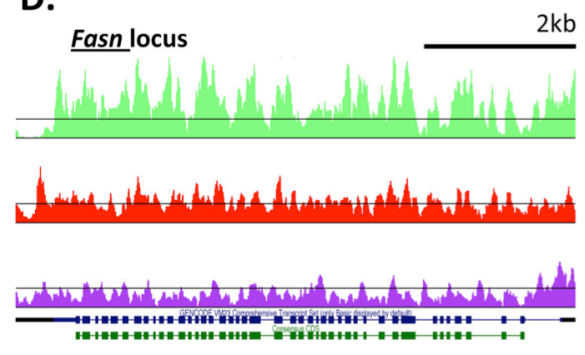

E.

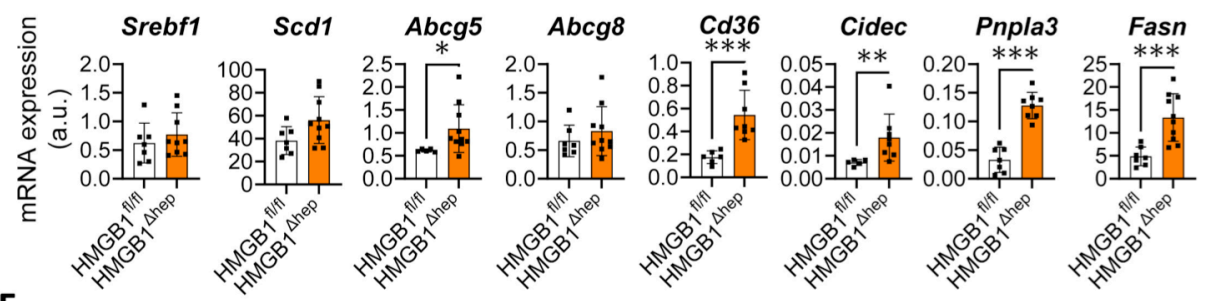

F.

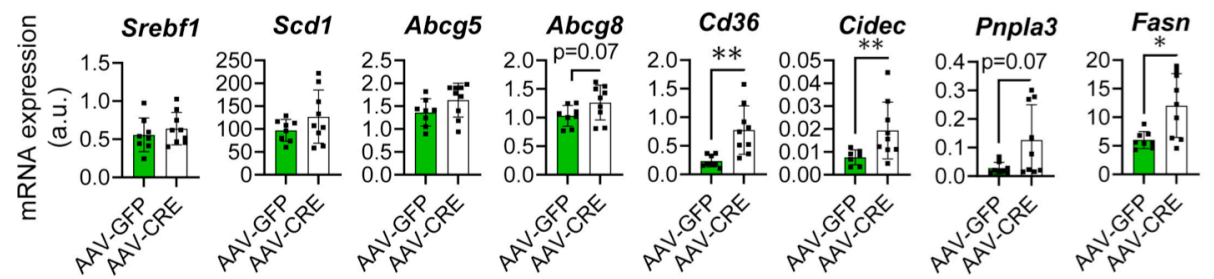

1240 Fig. 7. HMGB1 represses LXR $\alpha$ transcriptional activity in vitro.

1241 (A) Effect of HMGB1 on LXRE-luciferase reporter activity. Ad293 cells were treated with 1242 DMSO (vehicle), T0901317 (noted T09) $(0.1 \mathrm{uM})$ and/or LG286 (1 nM) for 14 hours. (B) Co1243 immunoprecipitation assay was performed to detect a potential interaction between HMGB1 and LXR in Ad293 transfected cells treated with DMSO (vehicle) or T0901317 (0.1 nM for 14 hours. Data are representative of three independent experiments. (C-D) Genome browser shot of ChIP-seq data along the locus of Acly and Fasn gene loci in liver from HMGB1 ${ }^{\mathrm{fl} / \mathrm{fl}}$ and HMGB1 ${ }^{\triangle \mathrm{Hep}}$ mice upon chow diet (green), HFD (purple) and after F/R (red). Gene (blue) and CDS (green) models are displayed on the bottom track. (E) Gene expression of direct (Srebfl, Scd-1, Abcg-5 and Abcg-8) and indirect (Cd-36, Cidec, Pnpla3 and Fasn) targets of LXR $\alpha$ in livers of $\mathrm{HMGB}^{\mathrm{fl} / \mathrm{fl}}(\mathrm{n}=7)$ and $\mathrm{HMGB}^{\Delta \mathrm{Hep}}(\mathrm{n}=9)$ mice. (F) Adult HMGB1 ${ }^{\mathrm{fl} / \mathrm{fl}}$ mice were infected either with AAV8-Gfp $(\mathrm{n}=8)$ or AAV8-TBG-Cre $(\mathrm{n}=9)$ to selectively generate Hmgbl deletion in hepatocytes in vivo and expression of direct (Srebf1, Scd-1, Abcg-5 and Abcg-8) and indirect (Cd-36, Cidec, Pnpla3 and Fasn) responsive genes were determined using RT-qPCR. Data are means \pm SEM of three independent experiments. $* p<0.05, * * p<0.01, * * * p<0.001$, $* * * * \mathrm{p}<0.0001$ by unpaired Mann and Whitney comparison or two-way ANOVA. $\$ \mathrm{p}<0.05, \mathbf{\$} \$$ $\mathrm{p}<0.01, \$ \$ \$ \mathrm{p}<0.001$, for treatment effect by two-way ANOVA. 